OPEN ACCESS

Edited by:

Paula Casati

Consejo Nacional de Investigaciones Cientificas y Técnicas (CONICET),

Argentina

Reviewed by:

Sharyn Elaine Perry, University of Kentucky, United States Kalika Prasad, Indian Institute of Science Education and Research, Thiruvananthapuram,

India

*Correspondence: Matgorzata D. Gaj mmdgaj@us.edu.pl

Specialty section

This article was submitted to Plant Genetics and Genomics, a section of the journal

Frontiers in Plant Science

Received: 29 May 2017 Accepted: 14 November 2017 Published: 11 December 2017

Citation:

Wójcik AM, Nodine MD and Gaj MD (2017) miR160 and miR166/165

Contribute to the LEC2-Mediated

Auxin Response Involved in the Somatic Embryogenesis Induction in Arabidopsis. Front. Plant Sci. 8:2024. doi: 10.3389/fpls.2017.02024

\section{miR160 and miR166/165 Contribute to the LEC2-Mediated Auxin Response Involved in the Somatic Embryogenesis Induction in Arabidopsis}

\author{
Anna M. Wójcik ${ }^{1}$, Michael D. Nodine ${ }^{2}$ and Małgorzata D. Gaj ${ }^{1 *}$ \\ ${ }^{1}$ Department of Genetics, University of Silesia, Faculty of Biology and Environmental Protection, Katowice, Poland, ${ }^{2}$ Gregor \\ Mendel Institute, Austrian Academy of Sciences, Vienna Biocenter (NBC), Vienna, Austria
}

MicroRNAs are non-coding small RNA molecules that are involved in the post-transcriptional regulation of the genes that control various developmental processes in plants, including zygotic embryogenesis (ZE). miRNAs are also believed to regulate somatic embryogenesis (SE), a counterpart of the ZE that is induced in vitro in plant somatic cells. However, the roles of specific miRNAs in the regulation of the genes involved in SE, in particular those encoding transcription factors (TFs) with an essential function during SE including $L E A F Y$ COTYLEDON2 (LEC2), remain mostly unknown. The aim of the study was to reveal the function of miR165/166 and miR160 in the LEC2-controlled pathway of SE that is induced in in vitro cultured Arabidopsis explants.In ZE, miR165/166 controls the PHABULOSA/PHAVOLUTA (PHB/PHV) genes, which are the positive regulators of $L E C 2$, while miR160 targets the AUXIN RESPONSE FACTORS (ARF10, ARF16, ARF17) that control the auxin signaling pathway, which plays key role in LEC2-mediated SE. We found that a deregulated expression/function of miR165/166 and miR160 resulted in a significant accumulation of auxin in the cultured explants and the spontaneous formation of somatic embryos. Our results show that miR165/166 might contribute to SE induction via targeting $P H B$, a positive regulator of LEC2 that controls embryogenic induction via activation of auxin biosynthesis pathway (Wójcikowska et al., 2013). Similar to miR165/166, miR160 was indicated to control SE induction through auxin-related pathways and the negative impact of miR160 on ARF10/ARF16/ARF17 was shown in an embryogenic culture. Altogether, the results suggest that the miR165/166- and miR160-node contribute to the LEC2-mediated auxin-related pathway of embryogenic transition that is induced in the somatic cells of Arabidopsis. A model summarizing the suggested regulatory interactions between the miR165/166-PHB and miR160-ARF10/ARF16/ARF17 nodes that control SE induction in Arabidopsis was proposed.

Keywords: AUXIN RESPONSE FACTOR10, ARF16, ARF17, LEAFY COTYLEDON2, miRNA, PHABULOSA, PHAVOLUTA, somatic embryogenesis 


\section{INTRODUCTION}

The unique developmental plasticity of plant cells has been widely documented by the successful formulation of in vitro culture protocols that enable the efficient clonal propagation of numerous plant species (reviewed Misra and Saema, 2016). In a vast number of these protocols, plants are regenerated via somatic embryogenesis (SE), a unique developmental process in which already differentiated somatic cells undergo embryogenic transitions giving rise to somatic embryo production (reviewed in Altamura et al., 2016). Thus, the identification of the genetic networks that govern SE induction greatly contributes to both the understanding of the molecular mechanisms that control plant totipotency and the improvement of the plant micropropagation protocols. In numerous plants including Arabidopsis thaliana (Arabidopsis), zygotic embryos at precisely defined developmental stages provide the most efficient tissue to induce SE (Elithi and Stasolla, 2011; Wójcikowska and Gaj, 2016). In Arabidopsis, the culture of zygotic embryos at a late cotyledonary stage of development on an auxin medium has been recommended to induce SE for molecular studies on plant cell totipotency (Gaj, 2004). Using this model SE system has resulted in the remarkable progress in deciphering the genetic mechanisms that govern SE induction that has been achieved in recent years (reviewed in Wójcikowska and Gaj, 2016).

A predominant number of genes with a documented decisive role in SE induction encode transcription factors (TFs) including, BABY BOOM (BBM) (Boutilier et al., 2002), WUSCHEL (WUS) (Zuo et al., 2002), AGAMOUS-LIKE15 (AGL15) (Harding et al., 2003) and LEAFY COTYLEDON (LEC1, LEC2) (Stone et al., 2001; Gaj et al., 2005) (reviewed in Nowak and Gaj, 2016). Global analysis of SE-transcriptome in Arabidopsis indicated that in concert with extensive modulation of TF genes (Gliwicka et al., 2013) numerous miRNAs are differentially expressed in embryogenic culture (Szyrajew et al., 2017). Similar to Arabidopsis, differential expression of miRNAs was reported in the embryogenic cultures of other plants, including Oryza sativa (Chen et al., 2011), hybrid yellow poplar (Li et al., 2012), Larix laptolerix (Zhang et al., 2012), Dimocarpus longan (Lin and Lai, 2013), Gossypium hirsutum (Yang et al., 2013) and Zea mays (Chávez-Hernández et al., 2015). Thus, it is believed that in control of SE, like in other plant developmental processes including zygotic embryogenesis (ZE) (Jones-Rhoades et al., 2006) miRNAs are involved but the genes targeted by specific miRNA and their function in the mechanism governing embryogenic transition is mostly unknown.

Hence, identifying the miRNAs that regulate SE, extend our knowledge about the regulatory pathways controlling embryogenic transitions in somatic plant cells. The LEC2 gene encodes a plant specific B3-domain TF (Harada, 2001) that is essential for SE induction. The expression level of LEC2 was reported to positively impact embryogenic transition in somatic cells of Arabidopsis in planta and in vitro (Stone et al., 2001; Ledwon and Gaj, 2009) and transcripts of LEC2 were found to accumulate in the explant cells undergoing SE induction in response to auxin treatment (Kurczynska et al., 2007; Ledwon and Gaj, 2011). The LEC2-mediated mechanism controlling SE induction involves the activation of the YUCCA genes that contribute to the auxin biosynthesis via tryptophan-dependent pathway (Wójcikowska et al., 2013). The upstream elements that regulate $L E C 2$ during SE induction remain unknown and among the candidates are PHABULOSA/PHAVOLUTA (PHB/PHV) proteins of the class III HOMEODOMAIN LEUCINE ZIPPER (HD-ZIP III) TF family that directly activate $L E C 2$ in vegetative development of Arabidopsis (Tang et al., 2012). Transcripts of HD-ZIP III genes are targeted by miR165/166 (Zhong and Ye, 2007). In Arabidopsis, two copies of the MIR165 and six of the MIR166 genes produce the mature miR165 and miR166 molecules that comprise the sequence of 21 nucleotides differing by one nucleotide (Reinhart et al., 2002). A role of miR165/166 in the regulation of $P H B$ and $P H V$ genes was revealed by biochemical and genetic analysis (Tang et al., 2003; Jung and Park, 2007) and the engagement of miR165/166-PHB/PHV in control of diverse developmental processes was indicated including radial pattering in shoots (McConnell et al., 2001), development of ovules (Sieber et al., 2004) and leaves (Mallory et al., 2004), xylem specification and differentiation of pericycle (Miyashima et al., 2011) and vascular tissues (Du and Wang, 2015). During ZE, $P H B$, and $P H V$ are repressed by miR165/166 to properly regulate early patterning of embryos and prevent precocious expression of differentiation-promoting TFs (Grigg et al., 2009; reviewed in Jia et al., 2014). The embryos of gain-of function phb-1d mutant carrying defective miR165/166-binding site in the $P H B$ gene have larger shoot meristem and radialized cotyledons (McConnell and Barton, 1998). Consistent with a phenotype of the phb-1d mutant embryos, the miR165/166PHB/PHV module was shown to contribute to the establishment of bilateral symmetry and the shoot apical meristem (SAM) during ZE (Prigge et al., 2005; Smith and Long, 2010).

The miR160-mediated repression of AUXIN RESPONSE FACTOR TFs (ARFs), including ARF10, ARF16, and ARF17 regulates several aspects of plant development. For example, miR160-directed repression of ARF10 and ARF16 regulates root cap, RAM and primary as well as lateral root development (Wang et al., 2005; Bustos-Sanmamed et al., 2013), floral organs and ovary patterning (Damodharan et al., 2016), and seed germination (Liu et al., 2007). Moreover, plants expressing miR160-resistant versions of ARF17 had altered expression of the early auxin responsive genes and defective embryo, root, vegetative and floral organ development (Mallory et al., 2005). In addition, miR160/ARF17 controls pollen wall formation (Yang et al., 2013), and male sterility (Shi et al., 2015).

Floral organs in carpels $(f o c)$ mutants in the $3^{\prime}$ region of the MIR160a gene have reduced miR160 levels and exhibit abnormal cell divisions in the root meristem precursors and suspensor of ZEs (Liu et al., 2010). The expression of ARF10, ARF16 and $A R F 17$ was distinctly affected in the foc mutant suggesting that miR160 controls zygotic embryo development through auxin signaling (Liu et al., 2010).

The differential expression of the MIR160 and MIRNA165/166 genes and their mature miR160 and miR166 products in the embryogenic culture of Arabidopsis (Szyrajew et al., 2017) motivated us to explore the function of miR165/166 and miR160 during SE induction. To this end, the candidate 
targets of miR165/miR166 (PHB/PHV) and miR160 (ARF10/ARF16/ARF17) were evaluated in SE culture of Arabidopsis with a disturbed expression/function of the studied genes. The relation between the expression level of miR165/166 and miR160, their candidate target genes and the embryogenic potential of the explants was investigated. Our results indicate the involvement of miR165/166- $P H B / P H V$ and miR160-ARF10/ARF16 nodes in regulation of SE. We postulate that miR165/166 and miR160 contribute to the embryogenic transition in Arabidopsis through the indirect impact on the LEC2 expression and modulation of the auxin biosynthesis in the explant tissue. The results expand our knowledge on the genetic regulation of SE induction and indicate the new components, miR160 and miR165/166, that operate in this auxin-related developmental pathway.

\section{RESULTS}

\section{Expression Level of $P H B$ and $P H V$ during SE in Relation to $\mathrm{miR} 165 / 166$}

To test the potential regulatory impact of miR165/166 on somatic embryogenesis (SE), the level of the candidate target transcripts, $P H B$ and $P H V$, was evaluated during embryogenic transition induced in Arabidopsis explants. RT-qPCR analyses indicated a significant increase of $P H B$ (up to 8 -fold) and $P H V$ (up to 4 -fold) transcripts at the early (5 d) and advanced (10 days) SE stages (Figure 1). We analyzed an SE culture of previously characterized STTM165/166 line with an abolished miR165/166 function (Yan et al., 2012) and found a strong accumulation (up to 50-fold) of $P H B$ and $P H V$ transcripts (Figure 1), which suggests a negative impact of miR165/166 on $P H B$ and $P H V$ expression in SE.

\section{Expression of PHB and miR166 Is Localized in SE-Involved Explant Parts}

The activity of $P H B$ promoter in the embryogenic culture was monitored with the use of the $p P H B:: G U S$ line. The analysis showed that in freshly isolated explants ( 0 day) the GUS signal was dispersed across explant and the strongest signal was detected along the adaxial side of the cotyledons (Figure 2A) that is involved in SE induction (Kurczynska et al., 2007). Further intensification of the GUS signal in the cotyledons was observed in the explant undergoing SE induction (5 and 10 days culture) but the $P H B$ expression signal was also detected in other explant parts including the root (5 days) and hypocotyl (10 days) that are not involved in SE (Figures 2B,C).

Whole mount RNA in situ hybridizations (WISH) with miR166-specific probes were used to examine the spatiotemporal localization of miR166 in explants induced toward SE. The freshly isolated explants (0 day) exhibited strong miR166 signal at the basal explant part including the hypocotyl and the root (Figure 2D). The pattern of miR166 was drastically changed in the explants cultured on SE induction medium and the intensive accumulation of miR166 at the shoot apical meristem (SAM) and its proximity was characteristic of the explants that

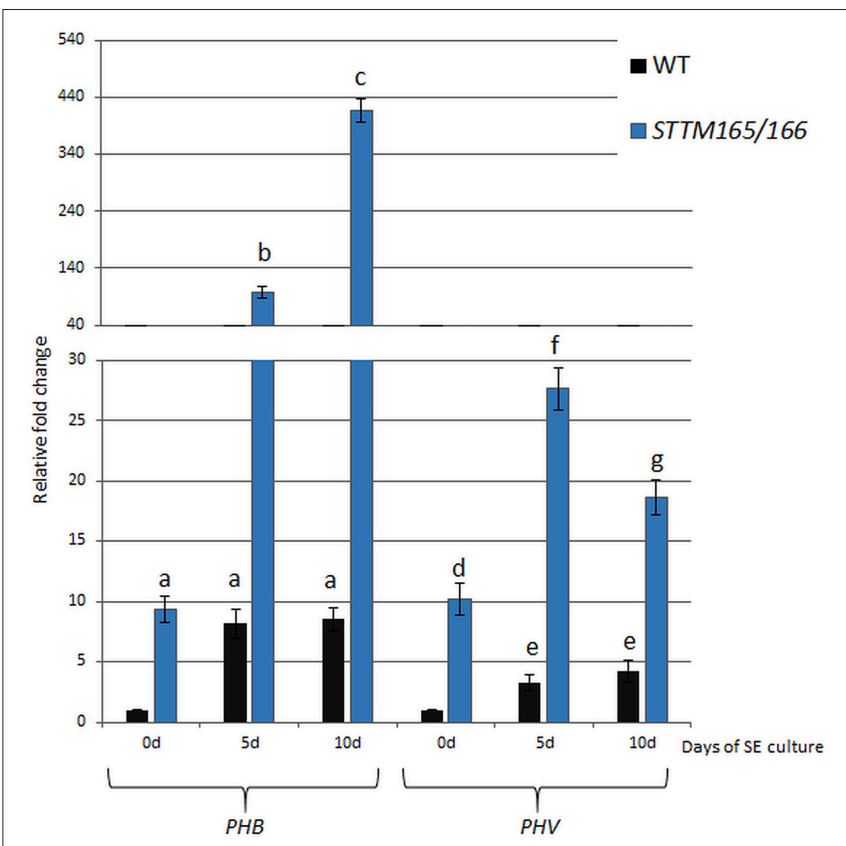

FIGURE 1 | Regulatory relation of miR165/166 and PHB/PHV in SE. Expression level of PHB and PHV in SE culture of WT (Col-0) and STTM165/166 transgenic line that were induced on medium with $5 \mu \mathrm{M} 2,4-\mathrm{D}$. Relative transcript level was normalized to the internal control (At4g27090) and calibrated to 0 day of the WT culture. Statistical analyses were performed using two-way ANOVA $(P<0.05)$ followed by Tukey's honestly significant difference test (Tukey HSD-test) $(P<0.05)$ in order to assess the differences between the gene expression at 0,5 , and 10 days of the SE culture within a genotype and between genotypes. Significantly different values are indicated by different letters $(P<0.05 ; n=3 \pm$ standard error) SE, somatic embryogenesis; d, day of SE culture.

were cultured for 5 days and at the SAM or cotyledons at the 10th day of SE (Figures 2E,F).

In order to verify the hypothesis that miR165/166 might negatively regulate the $P H B$ transcripts during SE induction, we compared the pattern of the GFP signal in $p P H B:: P H B-$ $G F P$ with the $P P H B:: m u P H B-G F P$ culture expressing $P H B$ transcripts that are resistant to the miR165/166 cleavage (Miyashima et al., 2011). Analysis of the GFP signal showed that the $P H B$ expression undergoes extensive spatio-temporal changes in the explants of both analyzed lines, but the pattern of $\mathrm{PHB}$ signal localization was distinctly different in 5 and 10 days culture of $p P H B:: m u P H B-G F P$ in comparison to $p P H B:: P H B-G F P$ (Figures 2G-I vs. Figures 2J-L) explants. In the $p P H B:: m u P H B-G F P$ tissue induced toward SE the PHB signal was detected in various explant parts including the hypocotyl, SAM and cotyledons (Figures 2H,I), while in the $P P H B:: P H B-G F P$ culture, the signal was limited to the cotyledons (Figures $\mathbf{2 K}, \mathbf{L}$ ). The apparent differences in the $P H B$ expression pattern in the $p P H B:: m u P H B-G F P$ vs. $p P H B:: P H B-G F P$ culture include a lack of $P H B$ expression in the hypocotyl and root part and less intensive GFP signals in the cotyledons of $p P H B:: P H B-G F P$ (Figures 2G-I vs. Figures 2J-L). 

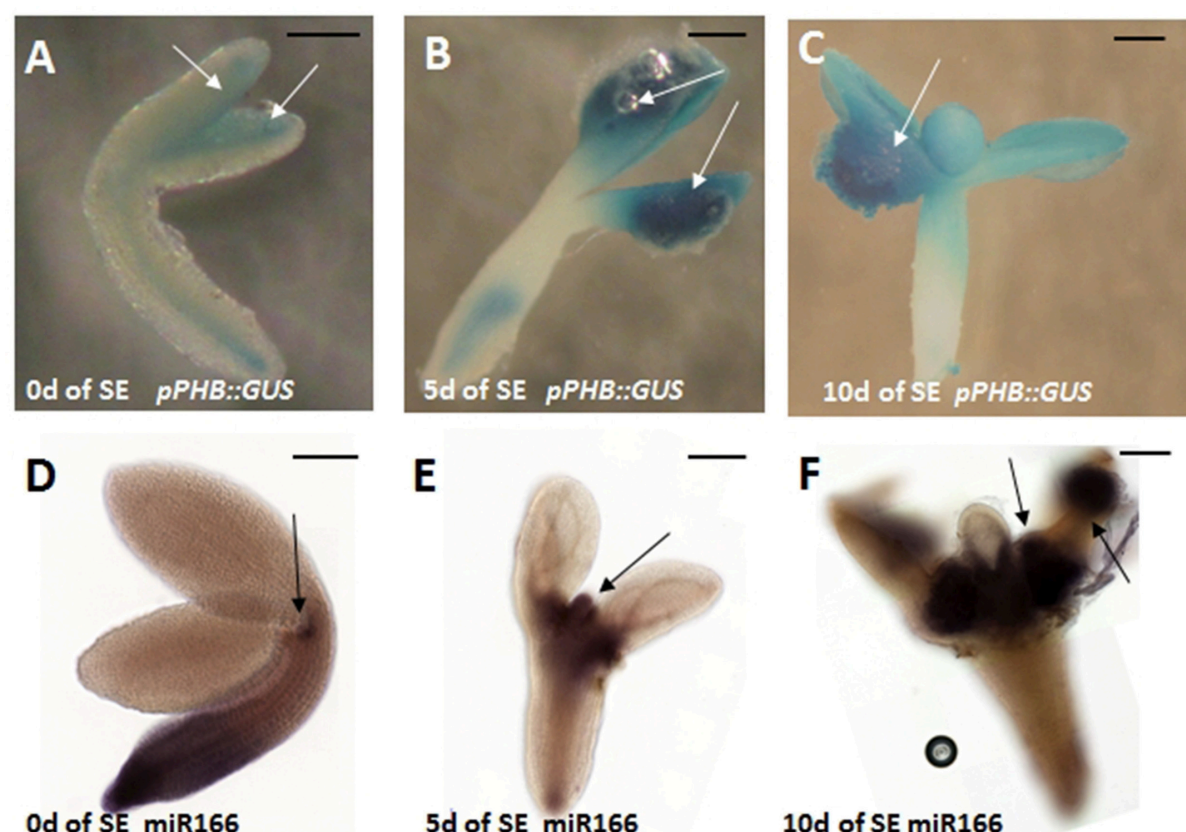

$\mathbf{E}$
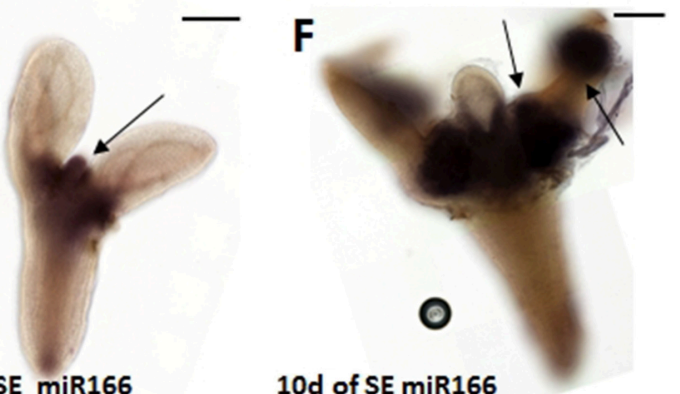

5d of SE miR166

10d of SE miR166
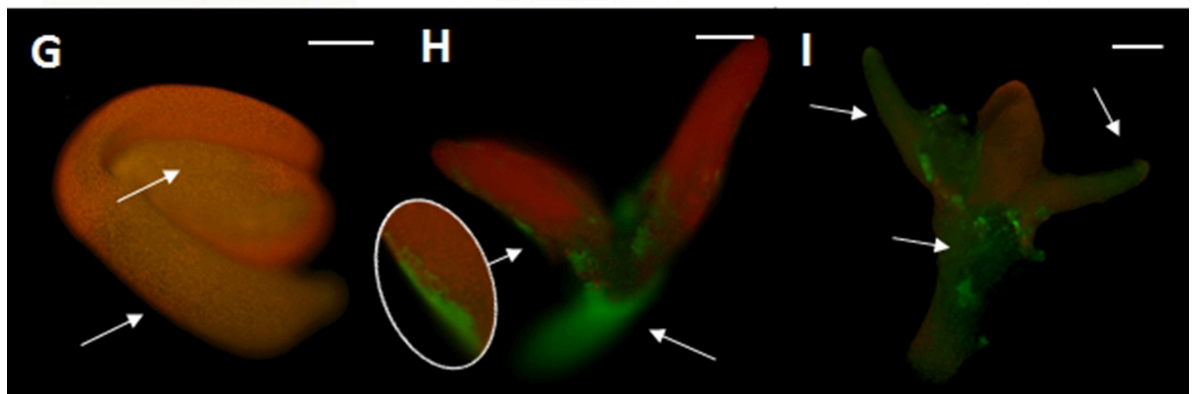

Od of SE pPHB::mUPHB-GFP

5d of SE PPHB::MUPHB-GFP

10d of SE PPHB::mUPHB-GFP
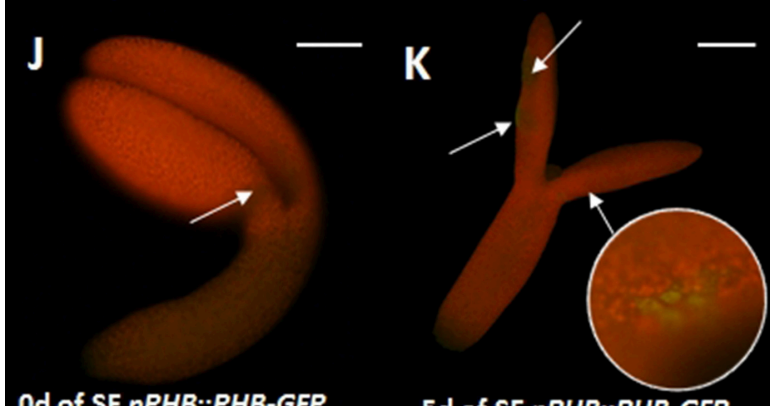

$\mathbf{L}$

Od of SE PPHB:PHB-GFP

5d of SE $p P H B * P H B-G F P$

10d of SE $P$ PHB::PHB-GFP

FIGURE 2 | Spatio-temporal expression pattern of miR166 and PHB in explants cultured on the SE induction medium with 5 $\mu \mathrm{M} 2,4-\mathrm{D}$ at 0, 5, and 10 days (0,5, 10 d). GUS- (A-C) and GFP-(G-L) monitored expression of PHB; WISH detection of miR166 (D-F). PHB expression in the pPHB::muPHB-GFP culture carrying a PHB transcript that is resistant to miR165/166 (G-I) miR165/166-restricted PHB expression in the pPHB::PHB-GFP culture (J-L). Arrows indicate the GUS/GFP signal associated with the SE-involved tissue. A probe against the mouse miR124 was used as the negative control (Figure S2). Scale bar indicate $100 \mu \mathrm{m}$. SE, somatic embryogenesis; d, day of SE culture.

\section{A Regulatory Relationship between miR165/166 and LEC2 in SE}

In order to investigate a potential relationship between miR165/166-PHB/PHV and LEC2 in SE we analyzed LEC2 expression levels in embryogenic cultures with disturbed expression of the miR165/166 and $P H B / P H V$ transcripts. We found that accumulation of the $P H B$ transcripts in the gainof-function phb1-d mutant and a STTM165/166 line led to the 
significantly increased LEC2 transcription (Figure 3A). Further evidence of a regulatory relationship between miR165/166$P H B / P H V$ and LEC2 was provided by the analysis of the embryogenic culture overexpressing LEC2 (Figure 3B). We observed that the overexpression of $L E C 2$ during SE resulted in a significantly increased level of the $P H B$ and $P H V$ transcripts, thus inferring a positive feedback loop between PHB/PHV and LEC2. In support of this postulate, we found that similar to $P H B$, also LEC2 is expressed at 5th and 10th day of culture in the cotyledons, i.e., the explant parts that are involved in SE induction (Figures 3C-E). Collectively, these observations support the hypothesis on a regulatory relation between miR165/166-PHB and LEC2 in the embryogenic transition.

Surprisingly, the up-regulation of LEC2 transcripts was also noticed in SE culture of the $p h b$ and $p h v$ insertional mutants with defected expression of $P H B$ (knock-out) and PHV (knockdown) genes, respectively (Figure 3A). To explain this result, we found the $P H B$ and $P H V$ transcripts to be up-regulated in $p h v$ and $p h b$ cultures, respectively (Figure S3). In conclusion, the level of the $P H B$ and $P H V$ transcripts appears to be controlled by a compensative regulatory mechanism in which insufficient expression of one of these genes results in a significantly increased transcription of the other gene.

\section{A Regulatory Relationship between miR160 and ARF10, ARF16, and ARF17 in SE}

To verify the assumption of the existence of a regulatory relationship between miR160 and ARF10/ARF16/ARF17 in SE, the cultures derived from $m i R 160 b$ and $m i R 160 c$ insertional lines were analyzed. The results indicated that the expression of ARF10 and ARF16 was up-regulated in the mutant cultures while the ARF17 transcription was down-regulated (Figure 4A). In addition, a significantly increased accumulation of the ARF16 transcripts was observed in cultures from the mARF16 line that carry the miR160-resistant form of ARF16 (Figure 4B). A negative feedback loop between ARF10/ARF16 and miR160 in SE might be suggested as we found an increased miR160 level in the cultures of $m A R F 16$ and arf10arf16 mutants (Figure 4C).
A

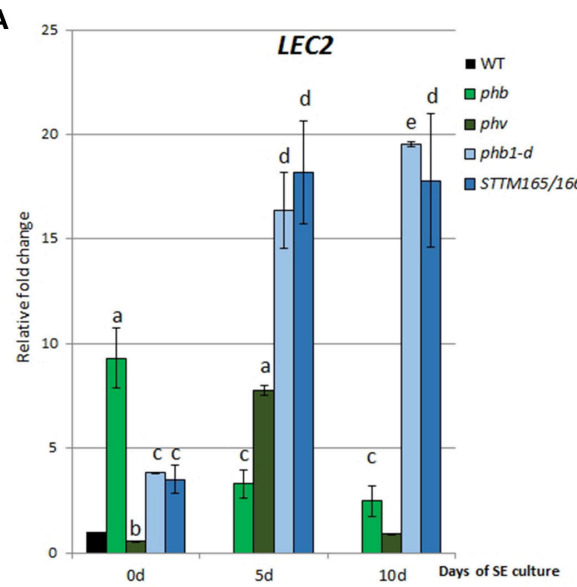

B

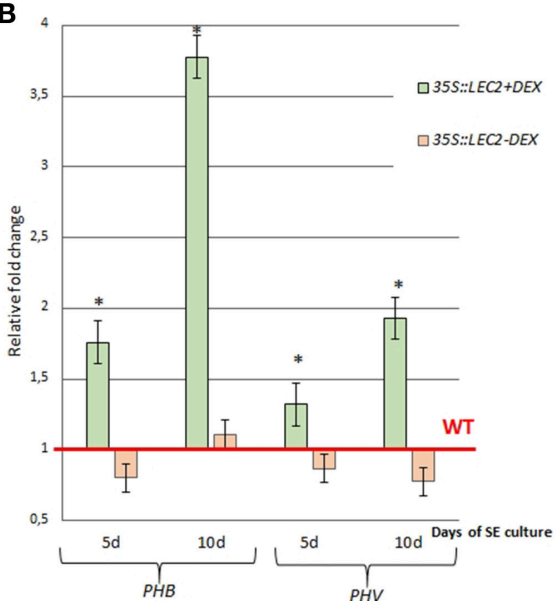

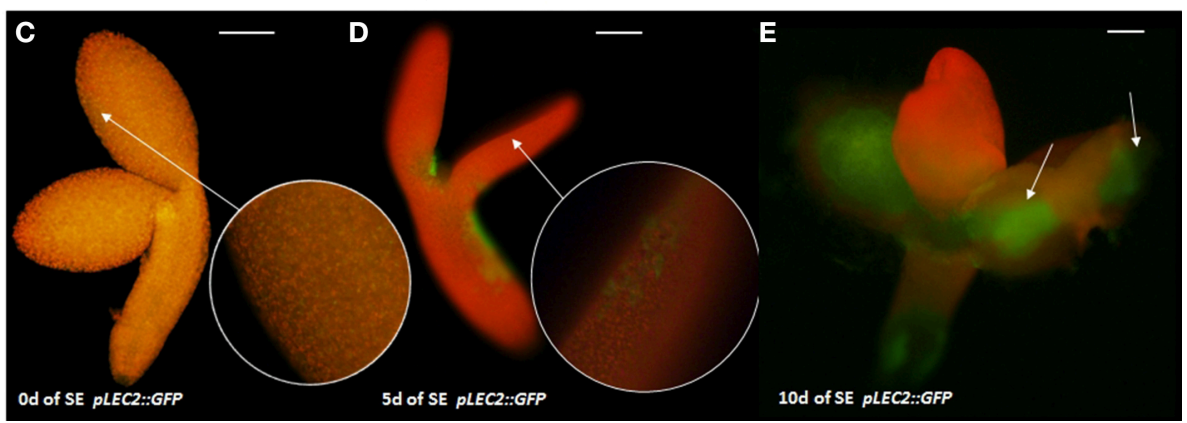

FIGURE 3 | Regulatory relation of miR165/166-PHB/PHV with LEC2 in the SE culture that was induced on the medium with $5 \mu M$ 2,4-D. (A) Expression level of $L E C 2$ in the SE culture of phb, phb1-d, phv, and STTM165/166 transgenic line. Relative transcript level was normalized to the internal control (At4g27090) and calibrated to 0 day of the WT culture. Statistical analyses were performed using two-way ANOVA $(P<0.05)$ followed by Tukey's honest significant difference test (Tukey HSD-test) $(P<0.05)$ in order to assess the differences between the gene expression at 0,5 , and 10 days of the SE culture within a genotype and between genotypes. Significantly different values are indicated by different letters $(P<0.05 ; n=3 \pm$ standard error) (B) Expression level of $P H B$ and $P H V$ in the SE culture of the DEX inducible 35S::LEC2-GR line on the medium with $(+)$ or without $(-)$ DEX. Statistical analyses were performed using the $T$-test $(p<0.05)$ to assess the differences between the genotypes. Values that were significantly different from the WT-derived culture of the same age are indicated with an asterisk ( $n=3 \pm$ standard error). (C-E) Localization of LEC2 expression in SE-induced pLEC2::GFP explants that were cultured for 0 (C), 5 (D), and 10 (E) days. Scale bar indicates $100 \mu \mathrm{m}$. SE, somatic embryogenesis; d, day of SE culture. Arrows indicate LEC2 expression in the explants areas undergoing SE induction. 
A

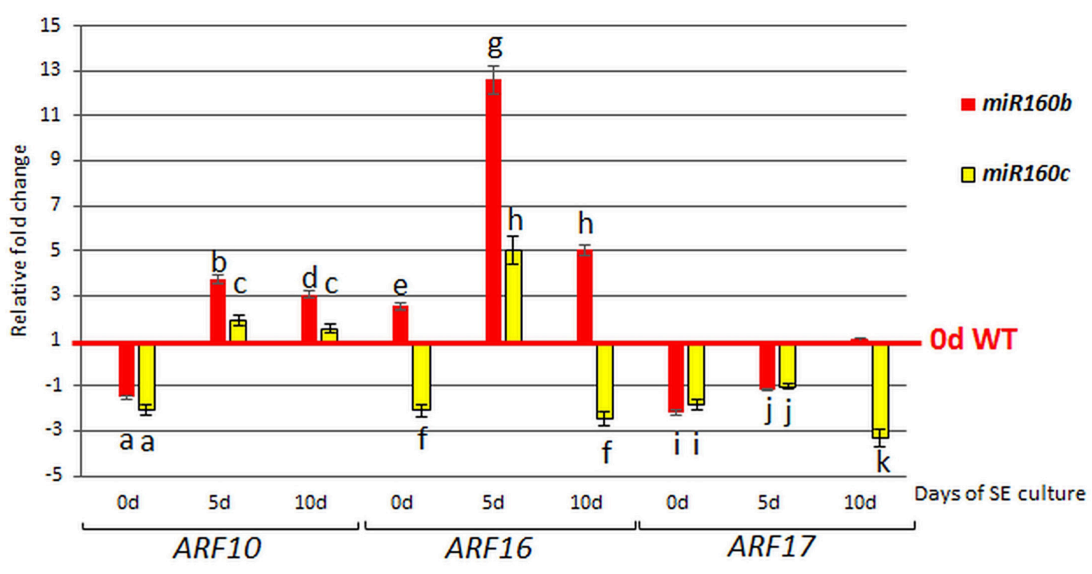

B

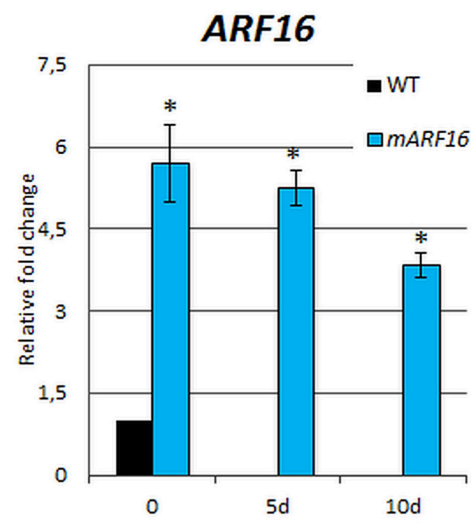

D

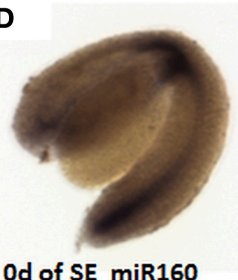

E

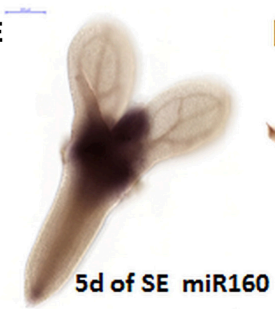

C

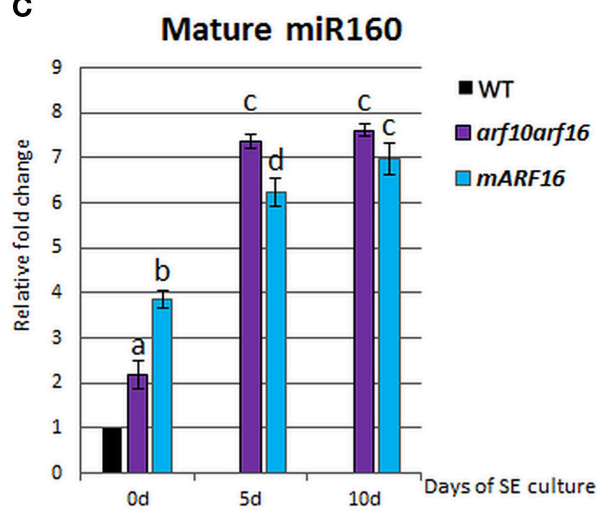

Mature miR160
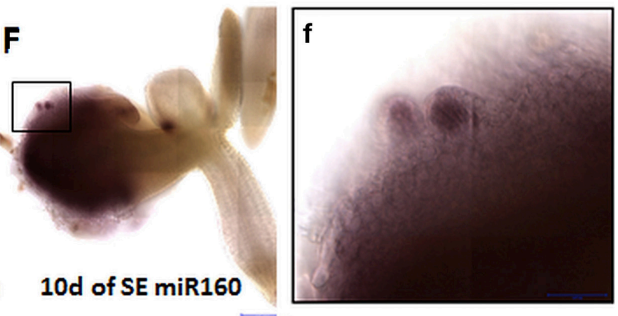

FIGURE 4 | Regulatory relation of miR160 and the target $A R F 10$, ARF16, and ARF17 genes in the SE culture that was induced on the medium with $5 \mu \mathrm{M} 2,4-\mathrm{D}$. Expression levels of ARF10, ARF16, and ARF17 in the miR160b and miR160c culture (A) ARF16 in the mARF16 culture (B) miR160 in the arf10arf16 and mARF16 cultures (C). WISH detection of miR160 in the explants that were induced toward SE at 0 (D), $5(\mathbf{E}), 10$ days $(\mathbf{F})$. A higher magnification (f) of an area marked in (F) showing an accumulation of miR160 in the somatic embryo. Relative transcript level was normalized to the internal control (At4g27090) and calibrated to the 0 day of the WT culture. Statistical analyses were performed using two-way ANOVA $(p<0.05)$ followed by Tukey's honest significant difference test (Tukey HSD-test) $(P<0.05)$ in order to assess the differences between gene expression at 0,5 , and 10 days of the SE culture within a genotype and between genotypes. Statistically significant differences $(P<0.05)$ are indicated by different letters $(P<0.05 ; n=3 \pm$ standard error). Statistical analyses (B) were performed using the $T$-test $(P<0.05)$ to assess the differences between the genotypes. Values that were significantly different from the WT-derived 0 day of the culture are indicated with an asterisk $(P<0.05 ; n=3$ \pm standard error). Probes against the mouse miRNA miR124 were used as the negative controls (Figure S2). SE, somatic embryogenesis; d, day of SE culture.

\section{WISH Localization of miR160 in SE}

WISH analysis with a miR160-specific probe indicated that miR160 localization patterns changed in explants during SE. More specifically, the explant at 0 day exhibited miR160 signal along a basal part of the explant while after 5 days of culture on the SE-induction medium, a strong accumulation of miR160 was observed in the SAM and its proximity (Figures $4 D-E$ ). At the advanced stage of SE (10 day) the miR160 signal was moved from the SAM area into the embryogenic tissue produced on cotyledons (Figure 4F) and a strong accumulation of miR160 was characteristic of somatic embryos (Figure $4 \mathbf{f}$ ).

\section{miR160-ARF10/16 and LEC2 Interact during SE}

To investigate a relation of miR160 with the LEC2-mediated pathway of SE induction, the expression level of LEC2 in the embryogenic cultures with impaired expression of miR160 (miR160b, miR160c) and its target genes, ARF10 and ARF16 
(mARF16, arf10arf16) was analyzed. LEC2 transcripts were accumulated in miR160b, miR160c, and mARF16 cultures and down-regulated in arf10arf16 culture (Figure 5A). Thus, a regulatory relationship between miR160-ARF10/ARF16 and LEC2 appears to be engaged in SE induction. In addition, a positive impact of $L E C 2$ on ARF10/ARF16 cannot be ruled out as we noticed the increased expression of ARF10 and ARF16 in the embryogenic culture with $L E C 2$ overexpression (Figure 5B). In contrast, $A R F 17$ transcripts was not affected in the LEC2overexpressing culture suggesting that LEC2 does not regulate ARF17 during SE.

\section{miR160 and miR165/166 Affect the Endogenous Auxin Content in the SE-Induced Explants}

The regulatory relationships of miR160 and miR165/166 with the LEC2 gene, a key regulator of SE through the control of auxin biosynthesis (Wójcikowska et al., 2013), suggested that the biological function of these miRNAs during SE might be related with auxin response. Consistent with this hypothesis, we observed that the embryogenic response that was displayed by the mutants with a disrupted expression and function of miR160 and miR165/166 on the auxin media was significantly different to the WT culture. That is, the miR160b, miR160c, $m A R F 16$, and STTM165/166 explants produced somatic embryos on the auxin-free medium and supplementation of the medium with 2,4-D drastically impaired their embryogenic response in a concentration-dependent manner (Figures 6A,B; Table S1). Thus, we hypothesized that miR160 and miR165/166 impact the auxin content in the SE-induced explants. In support of this hypothesis, we found that the levels of IAA-related indolic compounds (Bric et al., 1991) were significantly higher in the cultures of miR160b, miR160c, and STTM165/166 (Figure 6C). In addition, we found that the representative auxin-inducible AUX/IAA genes, IAA17, and IAA29 (Overvoorde et al., 2005), were up-regulated in the SE cultures with a reduced expression of miR160 (miR160b, miR160c) or an increased expression of the presumptive targets (STTM165/166, mARF16) (Figure S4). Because our results indicated that miR160 and miR165/166 control the LEC2 that has a regulatory role in the YUCCA (YUC)mediated pathway of auxin biosynthesis during SE (Wójcikowska et al., 2013), we profiled the expression of YUC genes in STTM165/166, mARF16, miR160b, and miR160c cultures. We found that YUC1, YUC4, and YUC10 genes were highly upregulated in these cultures (Figure 6D). Collectively, our results suggest that miR160 and miR165/166 may contribute to the embryogenic potential of Arabidopsis somatic tissues via the regulation of the LEC2-controlled pathway of auxin biosynthesis.

\section{Regulatory Relationship of miR160 and miR166/165 Pathways during SE}

Given that both miR160 and miR165/166 impact LEC2 expression during $\mathrm{SE}$ we then investigated the regulatory relationships between presumptive miR160 (ARF10, ARF16) and

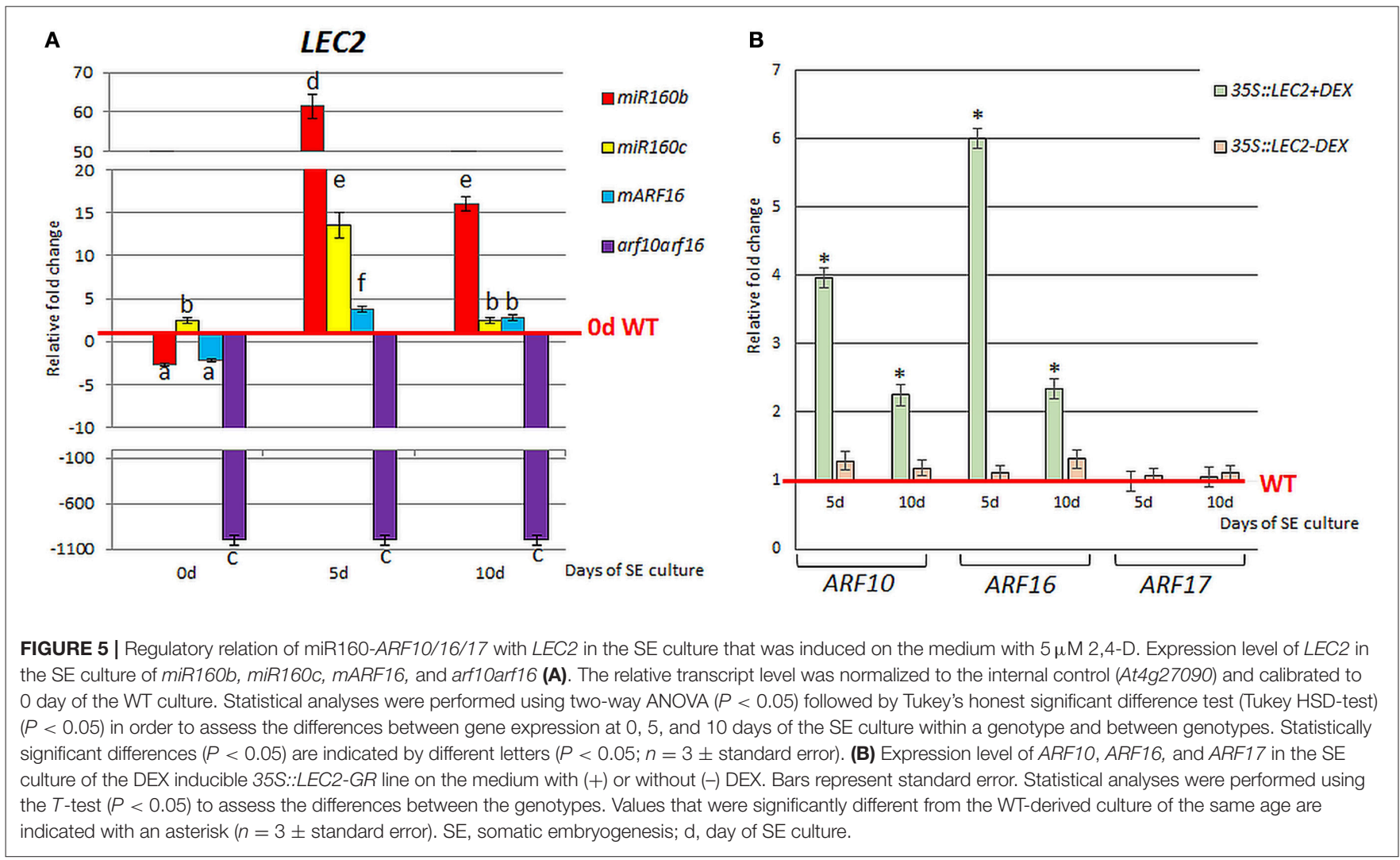


A

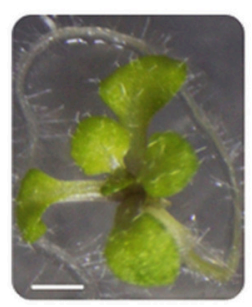

WT
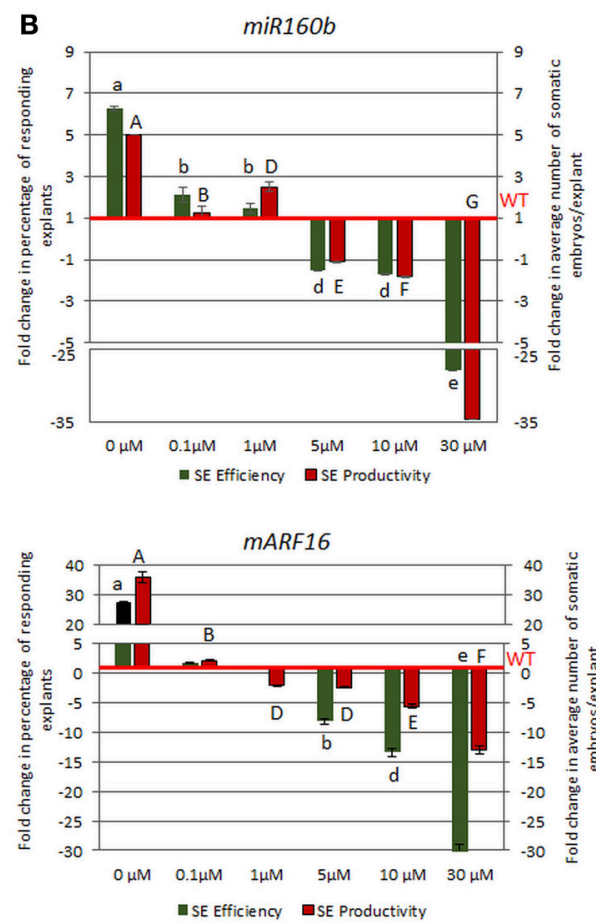

C

\begin{tabular}{lll} 
& TRANSGENIC LINE & $\begin{array}{c}\text { INDOLIC COMPOUNDS } \\
\text { ( } \mu \mathrm{g} / 1 \mathrm{~g} \text { of fresh tissue) }\end{array}$ \\
\hline $5^{\text {th }}$ day & Col-0 & $670,277 \pm 17,7$ \\
& miR160b & $757,84 \pm 25,5^{*}$ \\
& miR160c & $783,89 \pm 41,12^{*}$ \\
\hline $10^{\text {th }}$ day & Col-0 & $237,41 \pm 28,1$ \\
& miR160b & $256,86 \pm 13,4$ \\
& miR160c & $199,83 \pm 56,56$ \\
\hline $5^{\text {th }}$ day & Col-0 & $670,27 \pm 17,7$ \\
& STTM165/166 & $698,55 \pm 15,1$ \\
& mARF16 & $654,23 \pm 23,2$ \\
\hline \multirow{2}{*}{$0^{\text {th }}$ day } & Col-0 & $229,47 \pm 25,5$ \\
& STTM165/166 & $293,68 \pm 10,7^{*}$ \\
& mARF16 & $243,68 \pm 16,6$
\end{tabular}

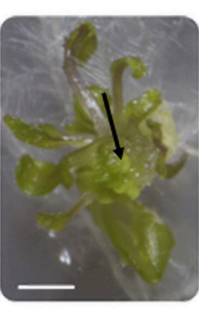

miR160b

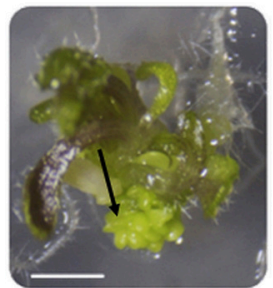

STTM165/166

miR160c
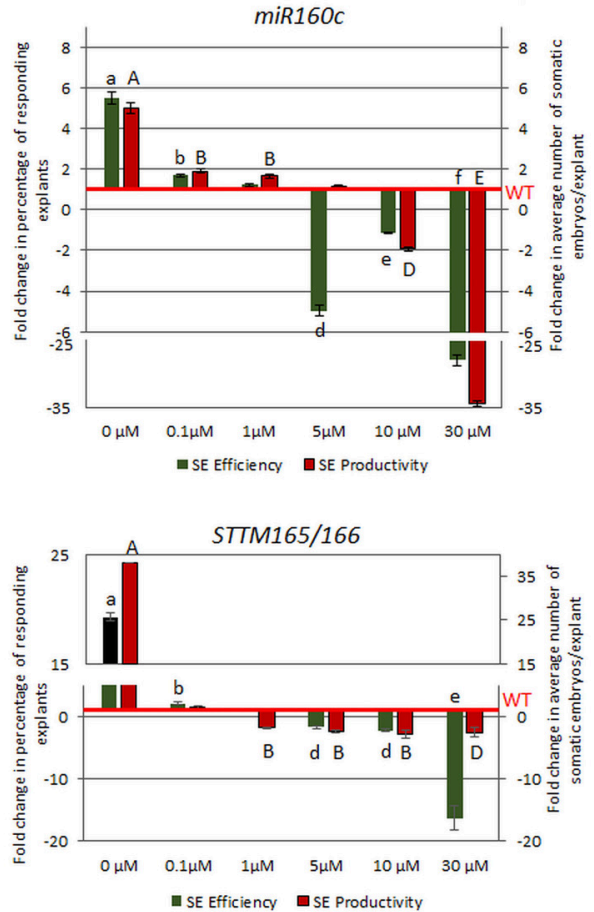

$\mathbf{D}_{2200}$
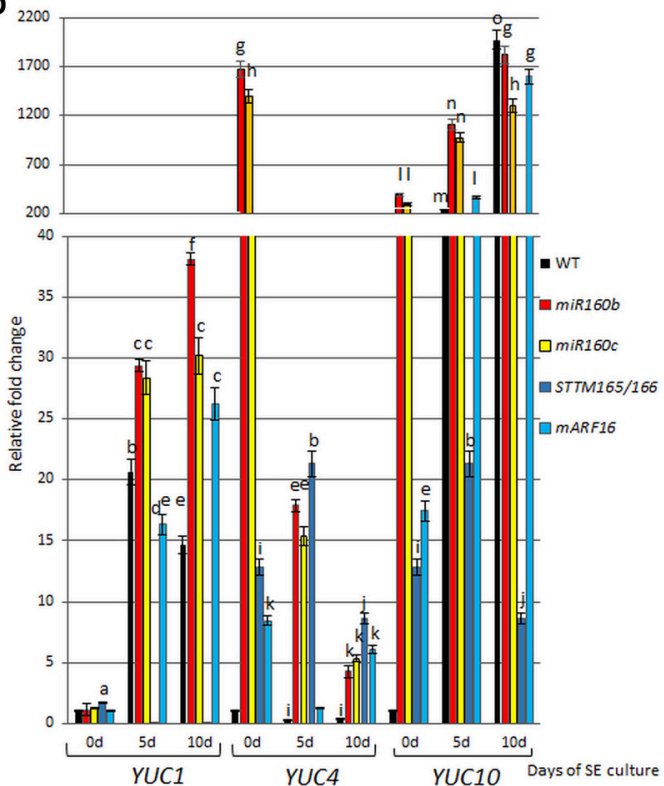

FIGURE 6 | Auxin-related function of miR160 and miR165/166 in the SE culture. The culture of miR160b, miR160c, mARF16, and STTM165/166 explants resulted in somatic embryo formation (marked with arrows) on the auxin-free medium (A) 2,4-D concentration-dependent embryogenic potential evaluated as the relative SE efficiency and productivity (B). SE efficiency and productivity was calibrated to WT culture. Statistical analyses were performed using two-way ANOVA $(P<0.05)$ 
FIGURE 6 | followed by Tukey's honest significant difference test (Tukey HSD-test) $(P<0.05)$ in order to assess the differences between the effect of different 2,4-D concentrations within a genotype and between genotypes. Statistically significant differences $(P<0.05)$ are indicated by different lower case letters (SE efficiency) or uppercase letters (SE productivity). Equivalent means have the same letter ( $P<0.05 ; n=3 \pm$ standard error). (C) The content of indolic compounds in the miR160b, miR160c, mARF16, and STTM165/166 explants during SE. Statistical analyses were performed using the $T$-test $(P<0.05)$ to assess the differences between the genotypes. Values that were significantly different from the WT-derived culture are indicated with asterisks $(P<0.05 ; n=3 \pm$ standard error). (D) Expression of $Y U C$ (YUC1, YUC4, and YUC10) genes that are involved in auxin biosynthesis in the miR160b, miR160c, mARF16, and STTM165/166 explants during SE. The relative transcript level was normalized to the internal control (At4g27090) and calibrated to the 0 day of the WT culture. Statistical analyses were performed using two-way ANOVA $(P<0.05)$ followed by Tukey's honest significant difference test (Tukey HSD-test) $(P<0.05)$ in order to assess the differences between gene expression at 0 , 5 , and 10 days of the SE culture and between genotypes. Statistically significant differences $(P<0.05)$ are indicated by different letters $(P<0.05 ; n=3 \pm$ standard error). Scale bar indicates $1 \mathrm{~cm}$. The SE culture (C,D) was induced on the medium with $5 \mu \mathrm{M} 2,4-D$.

miR165/166 (PHB/PHV) targets. We found that the level of $A R F 10$ and ARF16 transcripts was significantly increased in the phb, phv, phb1-d and STTM165/166 lines (Figure 7A), thus suggesting that $P H B$ might positively affect ARF10 and ARF16 expression. Moreover, $P H B$ was up-regulated in the culture of arf10arf16 double mutant, and down-regulated in mARF16, $m i R 160 b$ and miR160c indicating that ARF10 and ARF16 might negatively control $P H B$ expression (Figure 7B).

Altogether our results suggest that miR160 and miR165/166, possibly through the regulation of $A R F 10 / A R F 16$ and $P H B / P H V$, respectively, contribute to the $\mathrm{SE}$ induction mechanism associated with LEC2-controlled auxin biosynthesis pathway (Figure 8). However, further experiments are necessary to determine the mode of interaction between the miR160 and miR165/166 pathways during SE induction and to further elaborate the gene regulatory networks that they are involved in.

\section{MATERIALS AND METHODS}

\section{Plant Material}

The A. thaliana (L.) Heynh. Columbia (Col-0) and Landsberg erecta (Ler) parental genotypes and the transgenic lines miR160b, miR160c, phb, phv, phb1-d and pPHB::GUS were supplied by Nottingham Arabidopsis Stock Centre (NASC). The T-DNA insertional double mutants arf10-2 arf16-2 (hereafter noted as arf10arf16) and $P_{A R F 16}:$ mARF16 (hereafter noted $m A R F 16)$ were kindly provided by Prof. Xiaoya Chen (Institute of Plant Physiology and Ecology, China). The seeds of 2x35S::STTM165/166 line (hereafter noted as STTM165/166) were kindly provided by Dr. Guiliang Tang (Michigan Technological University, USA). The $p P H B:: P H B-G F P$ and pPHB:::muPHB-GFP line that carries the mutated, resistant to the miR165/166 cleavage version of the $P H B$ transcript (Miyashima et al., 2011), were kindly provided by Prof. Keiji Nakajima (Nara Institute of Science and Technology, Japan). The 35S::LEC2-GR line overexpressing $L E C 2$ upon DEX treatment was described previously (Ledwon and Gaj, 2009). To monitor expression of $L E C 2$ the $P L E C 2:: G F P$ line with nuclear localized GFP was generated by cloning the LEC2 promoter sequence $(-2020$ to +5 relative to ATG of $L E C 2$ ) into $\mathrm{pCR}^{\mathrm{TM}} 8 / \mathrm{GW} / \mathrm{TOPO} \mathbb{R}$ (Invitrogen), and then recombining these plasmids with Gateway-compatible pCGTAG plasmids using LR clonase (Invitrogen). Col-0 plants were transformed with use of the floral dip method (Clough and Bent, 1998).
The characteristics of the transgenic lines that were used in the study are presented in Table S2A.

\section{Plant Growth Conditions}

Seeds sterilized with $20 \%$ solution of commercial bleach were plated on $0.5 \mathrm{x}$ MS solid medium. The plates were kept at $4^{\circ} \mathrm{C}$ in darkness for 2 days and then transferred to a growth chamber at $21 \pm 1^{\circ} \mathrm{C}$ under a $16 / 8 \mathrm{~h}$ photoperiod of $40 \mu \mathrm{M} \mathrm{m}^{-2} \mathrm{~s}^{-1}$ white, fluorescent light. The young seedlings were transplanted into Jiffy-7 pots and grown in a walk-in type green room under the conditions described above until harvesting of siliques.

\section{Somatic Embryogenesis Induction}

Immature zygotic embryos (IZEs) at green cotyledonary-stage were used as explants to induce SE. IZEs were cultured in E5 solid medium with $5 \mu \mathrm{M}$ 2,4-dichlorophenoxyacetic acid (2,4-D) according to Gaj (2001). In some experiments (Figures 4A,B), other concentrations of 2,4-D were used including $0 ; 0.1 ; 1.0$; $10.0 ; 30.0 \mu \mathrm{M}$. The explant capacity for SE was evaluated in 21 day-culture and two parameters were calculated: SE efficiency (the percentage of explants that formed somatic embryos) and SE productivity (the average number of somatic embryos per explant). All of the culture combinations were evaluated in three replicates and at least 30 explants (ten explants/Petri dish) were analyzed per one replicate.

\section{Whole Mount in Situ Hybridization of miRNAs}

The whole mount in situ hybridization (WISH) of miRNA molecules was performed according to modified protocol of Dastidar et al. (2016). Embryos/explants were dissected/collected in a drop of PBS (Phosphate-buffered saline buffer) and immediately transferred to $4 \%$ paraformaldehyde on ice. LNA Digoxin 5'-end labeled probes were used following to the Dastidar et al. (2016). The slides were scanned using Panoramic FLASH 250 II.

\section{RNA Isolation and RT-qPCR Analysis}

An RNAqueous kit (Ambion) was used to isolate total RNA and mirVana ${ }^{\mathrm{TM}}$ Kit was used to isolate miRNAs from the IZE explants. Depending on the age of a culture, 300 ( 0 day culture) to 100 (10 days culture) explants were collected for RNA/miRNA isolation. The concentration and purity of RNA was evaluated with a ND-1000 spectrophotometer (Nano-Drop). To avoid DNA contamination, RNA was treated with RQ1 RNase-free DNase I (Promega) following the manufacturer's instructions. 
A

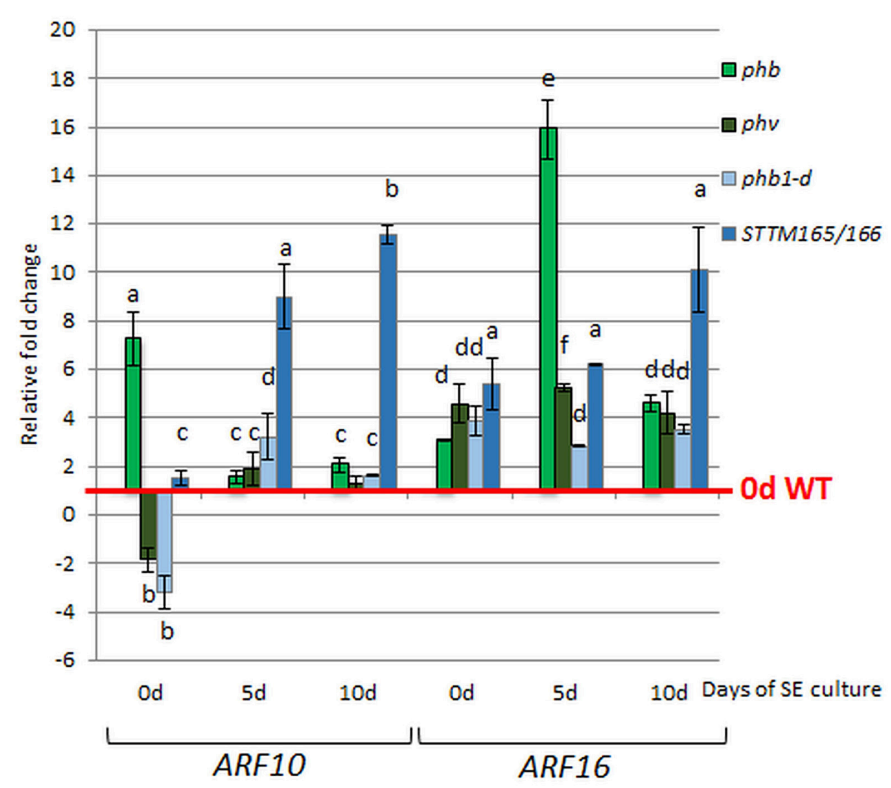

B $\quad$ PHB

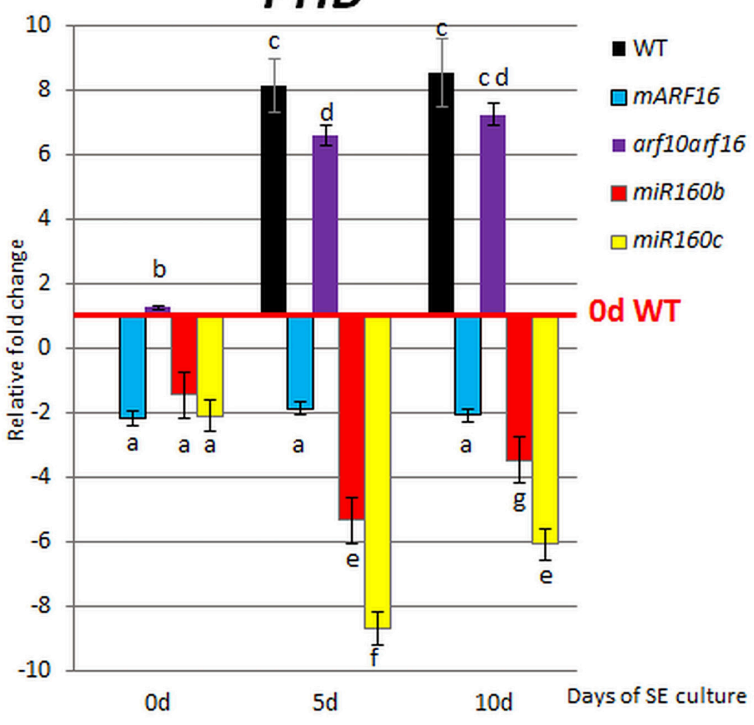

FIGURE 7 | Regulatory relationship of miR165/166 and miR160 nodules. Expression level of ARF10 and ARF16 in phb, phv, phb1-d, and STTM165/166 (A) and PHB in $m A R F 16$, arf10arf16, miR160b, and miR160c (B) SE culture that was induced on the medium with $5 \mu \mathrm{M} 2,4-\mathrm{D}$. The relative transcript level was normalized to the internal control (At4g27090) and calibrated to the 0 day of the WT culture. Statistical analyses were performed using two-way ANOVA ( $P<0.05)$ followed by Tukey's honest significant difference test (Tukey HSD-test) $(P<0.05)$ in order to assess the differences between gene expression at 0,5 , and 10 days of the SE culture within a genotype and between genotypes. Statistically significant differences $(P<0.05)$ are indicated by different letters $(P<0.05$; $n=3 \pm$ standard error).

First strand cDNA was produced using a RevertAid First Strand cDNA Synthesis Kit (Fermentas). RT-qPCR was carried out in a $10 \mathrm{~L}^{-1}$ reaction volume using a LightCycler 480 SYBR Green I Master (Roche). The primers that were relevant to the genes being studied were used in the RT-qPCR analysis (Table S2B). The RT-qPCR reactions were performed as previously described in Wójcik and Gaj (2016). Primary data analysis was performed using LightCycler Software (Roche).

\section{Detection of Mature miRNA}

The oligonucleotides design, stem-loop RT and real time qPCR were performed according to Speth and Laubinger (2014). The primers sequences used in the study are listed in Table S2B. Primary data analysis was performed using LightCycler Software (Roche). Relative RNA levels were calculated and normalized to an internal control of the At4g27090 gene encoded 60S ribosomal protein (Thellin and Zorzi, 1999). In all of the analyzed tissue samples, the control gene displayed a constant expression pattern with $\mathrm{Cp}=19 \pm 1$. The plant tissues for the analysis of gene expression were produced in three biological replicates and two technical replicates of each repetition were carried out. The relative expression level was calculated using $2^{-\Delta \Delta \mathrm{Ct}}$.

\section{Histological Analysis}

To detect GUS signal the $p P H B:: G U S$ explants were stained with a standard X-Gluc (Sigma Aldrich) solution at $37^{\circ} \mathrm{C}$ for $12 \mathrm{~h}$ according to Jefferson et al. (1987). The tissue was inspected under Delta-Optical SZ-630T microscope and images were saved as jpg files with a Canon EOS 60D camera.

Analysis of GFP signal was carried out using a Nikon Eclipse $\mathrm{Ni}-\mathrm{E} / \mathrm{Ni}-\mathrm{U}$ fluorescent microscope system. GFP fluorescence was excited with halogen lamphouses with a 100-240 VAC (Prior Lumen200) and a wavelength of $488 \mathrm{~nm}$. Photographic documentation was recorded by Nikon Digital Sight DS-Fi2 with DS-U3 camera, using the NIS-Elements F computer program version 4.0 .

\section{Evaluation of Indolic Compounds Level}

A colourimetric technique that enabled the detection of indolic compounds, including IAA, was applied (Bric et al., 1991; Wójcikowska et al., 2013). To evaluate the relationship between IAA content and miR160 and miR166 activity, IZEderived cultures of Col-0 and miR160b, mARF16, STTM165/166 genotypes were analyzed. IZE explants were induced on a $5 \mu \mathrm{M}$ $2,4-\mathrm{D}$ medium and tissues were sampled on the 5 th or 10 th day of culture. The procedure was performed as described previously (Wójcikowska et al., 2013). Each analysis was carried out in three biological replicates.

\section{Statistical Analysis}

The statistical analyses were performed using either the Student $t$-test or a two-way ANOVA $(p<0.05)$ followed by Tukey's honestly significant difference test (Tukey HSD-test) $(p<0.05)$. The figures show the averages from at least three biological replicates with the standard error. 


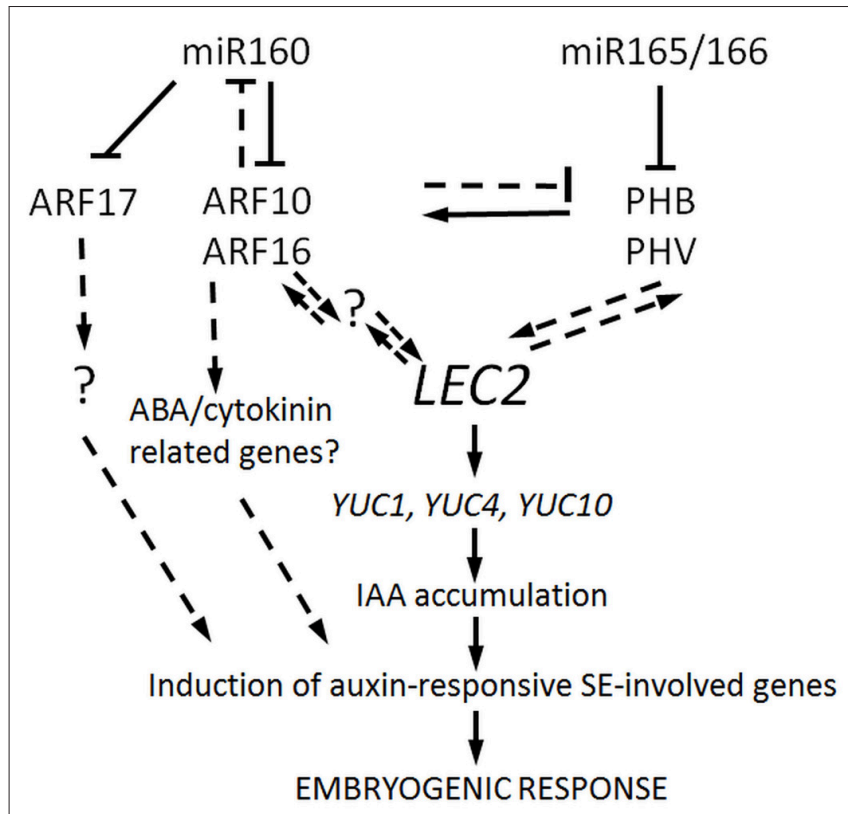

FIGURE 8 | A model of miR160- and miR165/166-regulated pathways contributing to SE induction. ARF10/ARF16 and PHB/PHV that are repressed by miR160 and miR165/166, respectively, are postulated to positively regulate LEC2, thus controlling SE induction via the up-regulation of YUC genes (YUC1,4, and 10) and the activation of the auxin biosynthesis pathway. As a consequence of IAA accumulation, the auxin-responsive genes that are involved in SE induction are triggered. The targets of miR160 and miR165/166 appear to interact via a negative feedback loop as PHB/PHV seems to positively control their repressors, ARF10/ARF16. Besides controlling the auxin response, ARF10/ARF16 might also contribute to SE induction by impacting the signaling pathways of other hormones including ABA and/or cytokines. The role of ARF17 in SE induction appears to be unrelated to the LEC2-mediated pathway. Further analyses are required to validate the assumed intra- and inter-specific regulatory interactions, which are indicated with dashed lines and to identify any other genetic components that are involved and are marked with a question mark.

\section{DISCUSSION}

Experimental and technological advances have recently provided evidence that in animals and plants dynamically fine-tuned expression of TRANSCRIPTION FACTORS (TFs) accounts for the correct pattern formation in the embryo developing from the zygotic cell (Jeong et al., 2012; Bedzhov et al., 2014). In embryogenic cultures of Arabidopsis the extensive changes in the transcriptomes of SE-induced cells that involve the profound modulation of both the TF transcripts and MIRNA/miRNA were indicated (Le et al., 2010; Xiang et al., 2011; Lara-Chavez et al., 2012; Gliwicka et al., 2013; Seefried et al., 2014; Szyrajew et al., 2017). Thus, it is expected that in SE, similarly to zygotic embryogenesis (ZE) (Nodine and Bartel, 2010), the miRNA-directed transcriptional regulation of the embryonic TFs might play the essential function. Consistent with this postulate, we studied the functions of miR160 and miR165/166 in embryogenic culture of Arabidopsis.

\section{miR165/166 Contributes to the SE Induction via PHB/PHV Regulation}

During ZE, miR165/166-mediated repression of the $P H B$ and $P H V$ genes enables the morphogenesis-to-maturation transition and correct specification of shoot apical meristem (SAM) and root apical meristem (RAM) in the developing embryo (Grigg et al., 2009; Miyashima et al., 2013). Relevant to zygotic development, our results suggest that miR165/166 restricts PHB/PHV expression to control embryonic development in Arabidopsis somatic cells cultured in vitro. In support of this postulate, we found that the expression profile of $P H B / P H V$ is inverse to the one that was indicated for miR165/166 in the WT embryogenic culture (Szyrajew et al., 2017) (Figure S1A). In line with this finding, we showed the $P H B$ and $P H V$ transcripts to be accumulated in the culture of the STTM165/166 lines with an abolished miR165/166 function. In order to further test the regulatory relationship between miR165/166 and $P H B$, the spatio-temporal patterns of $P H B$ and miR166 were investigated in SE-induced explants with use of reporter lines ( $p P H B:: G U S ; p P H B:: P H B-G F P$ ) and WISH, respectively. Our results indicated changes in the localization of PHB signal and miR166 during SE induction. Although PHB and miR166 both accumulate in the proximity of the shoot apical meristem (SAM), the area that is involved in SE induction (Kurczynska et al., 2007), their expression patterns appear to not overlap. Accordingly, cotyledons that are predominantly involved in somatic embryo formation displayed a strong expression of PHB while the WISH signal of miR166 was limited to the SAM and its proximity. Further evidence that miR166 might spatio-temporally repress the $P H B$ transcripts in SE-induced tissue was supported by the observation of a more widespread and intense $P H B$ expression in the $P P H B:: m u P H B-G F P$ than in the $p P H B:: P H B-G F P$ culture (Figures 2G-I vs. Figures 2J-L). miR165/166 appears to spatiotemporally restrict the $P H B$ transcripts in the explant parts/cells that are not responsive to SE induction. In contrast, the SE-responsive cells that are dispersed along the explant cotyledons (Kurczynska et al., 2007) seem to accumulate $P H B$ transcripts (Figures 1, 2K, L), which in part, might result from the decreased content of miR166 (Szyrajew et al., 2017). However, more advanced cytohistological analysis is required to evaluate the relation between the miR165/166 content and the $P H B$ transcript level in the explant cells that are undergoing SE induction.

Moreover, consistent with the finding that the $P$ PHB::PHBmuGFP plants phenocopy the miR165/166-resistant phb1-d mutant (Miyashima et al., 2013), we found that the pPHB:muPHBGFP line showed a reduced SE response, which was similar to the phb1-d mutant (Figure S5). The impaired SE response of pPHB:muPHB-GFP and phb1-d explants on an auxin medium that is standard for SE-induction is possibly caused by an increased IAA accumulation, which might result from the upregulation of LEC2 (Figure 3A) and the activation of auxin biosynthesis YUC genes (Figure 6D). A relation between the endogenous auxin accumulation and the impaired embryogenic potential of tissue on a standard auxin medium was indicated for different genotypes in the present (Figure 6B) and other studies 
(Wójcikowska et al., 2013; Wójcik and Gaj, 2016). Altogether, the analysis of the reporter lines suggests that in SE-induced explants miR166 might restrict $P H B$ expression to the cotyledon tissue and the resulting spatial distribution of $P H B$ expression colocalizes with explant sites developing somatic embryos.

The rapid and intense accumulation of $P H B$ transcripts in SE-involved explant parts that we observed in response to SE induction medium suggests that $\mathrm{PHB}$ might be involved in the very early events associated with the embryogenic transition of somatic cells. The genetic regulation of early events associated with embryogenic transition in the in vitro cultured somatic cells are poorly known and identification of the genes acting upand down-stream from $P H B$ in the very early SE culture might provide new insights into molecular mechanism of SE induction.

\section{miR160 Impacts SE via Control of Auxin Signaling Regulators, ARF10/ARF16/ARF17}

Recently, the impact of miR160 on developmental processes induced in vitro was reported. Accordingly, miR160-mediated repression of ARF10/ARF16/ARF17 was postulated to control the embryogenic response in culture of $D$. longan (Lin et al., 2015) and miR160-ARF10 was shown to control cellular reprogramming and callus formation in Arabidopsis (Liu et al., 2016). Our results indicate that miR160 might also contribute to $\mathrm{SE}$ induction in Arabidopsis through the repression of ARF10, $A R F 16$, and $A R F 17$, in light of (i) the opposite expression patterns of ARF10/ARF16/ARF17 and miR160 in the Arabidopsis embryogenic culture (Szyrajew et al., 2017; Wójcikowska and Gaj, 2017) (Figure S1B); (ii) the increased expression of $A R F 10$ and ARF16 in the miR160 mutant cultures and the accumulation of ARF16 in the cultures expressing the miRNA-resistant version of ARF16. Differences in the accumulation of ARF10/ARF16 at distinct time points of the miR160 mutants SE culture may be caused by a redundancy between the members of the MIR160 family, all of which are able to cleave transcripts of $A R F 10$ and ARF16 (http://plantgrn.noble.org/psRNATarget/).

The unimpaired SE response in arf10 and arf16 single mutants compared to the impaired SE response of double arf10arf16 mutants (Figure S5) suggests that ARF10 and ARF16 seem to function redundantly during SE. In support of this postulate, the redundant function of ARF10 and ARF16 was indicated in root cap formation (Wang et al., 2005) and seed dormancy (Liu et al., 2013) and recently, ARF10/ARF16 together with IAA17 were found to act as a protein complex (Ye et al., 2016).

Interestingly, we observed the significant accumulation of miR160 in $m A R F 16$ and arf10arf16 cultures that suggests a role of ARF16 in the control of miR160 during SE. The feedback regulation of miRNA production by the associated target genes was reported for mammalian and plant miRNAs (Wu et al., 2009; Lai et al., 2016). Importantly for the present result, miR160 was found to be negatively regulated by the targeted ARF17 to control auxin homeostasis and adventitious rooting in Arabidopsis (Gutierrez et al., 2009). Thus, it cannot be excluded that during SE in Arabidopsis miR160 is controlled by the targeted ARFs but this assumption needs further experimental verification.
The distinct differences in the level of the primary transcripts of MIR160a, MIR160b, and MIR160c in Arabidopsis embryogenic culture (Szyrajew et al., 2017) suggest that MIR160 genes contribute differently to the regulation of SE. In line with this assumption we found $A R F 10$ and $A R F 16$ but not $A R F 17$ to be accumulated in miR160b and miR160c mutant cultures. This result implies that during SE ARF17 might be under the control of MIR160a. In support for this postulate, exclusively MIR160a was indicated to control ARF17 during early development of zygotic embryo (Liu et al., 2010). The analysis of ARF17 expression in the SE culture with defected MIR160a expression would verify this hypothesis but the severely reduced fertility of the foc mutant (miR160a) (Liu et al., 2010) makes isolation of immature zygotic embryos and thus the establishment of SE culture difficult.

The assumption that a role of ARF17 in SE might be different to ARF10/ARF16 supports also the finding that in embryogenic culture expression of $A R F 17$, in contrast to ARF10/ARF16, is not affected by $L E C 2$ overexpression. Moreover, the expression pattern of ARF17 in SE differs from ARF10/ARF16 and accordingly, a level of ARF17 mRNAs increases in late SE and is auxin-independent (Wójcikowska and Gaj, 2017). Thus, it cannot be ruled out that miR160-ARF17 operates in the advanced SE culture associated with the formation of somatic embryos. Suggestive for this postulate is formation of the defective zygotic embryos in plants with suppressed miR160-directed regulation of ARF17 (Mallory et al., 2005).

\section{miR160 and miR165/166 Control SE Induction via LEC2-Stimulated Pathway of Auxin Biosynthesis}

Importantly for a role of miR160- and miR165/166-regulated pathways in SE, we found that transgenic forms with defective expression and function of miR160 (miR160b, miR160c, mARF16) and miR165/166 (STTM165/166) were capable to produce somatic embryos on auxin-free medium and auxin treatment severely impaired their embryogenic response. Similar capacity for SE induction on auxin-free medium displayed also the culture overexpressing LEC2 and accumulated IAA (Ledwon and Gaj, 2011; Wójcikowska et al., 2013). Similar to the culture overexpressing $L E C 2$, in embryogenic culture of the miR160 and STTM165/166 we found increased accumulation of the indolic compounds and enhanced expression of $L E C 2$ that was coupled with activation of the YUC (YUC1, YUC4, and YUC10) genes encoding the auxin biosynthesis enzymes involved in SE induction in Arabidopsis (Wójcikowska et al., 2013).

The results imply that miR165/166 might control SE induction by impacting the $P H B$ and $P H V$ genes that encode the direct activators of LEC2 (Tang et al., 2012). Although the PHB and PHV are closely related and demonstrate the high degree of functional interchangeability (McConnell et al., 2001; Prigge et al., 2005) the mechanism adjusting their individual contribution to the controlled processes, including SE, has not been yet revealed.

In support for the LEC2-related function of PHB in SE we observed the up-regulated expression of $L E C 2$ in embryogenic 
culture of phb-1d mutant that was reported to spontaneously produce somatic embryos (Tang et al., 2012). Moreover, we found LEC2 overexpression to enhance $P H B / P H V$ transcription level suggesting that a positive feedback regulation exists between $P H B / P H V$ and $L E C 2$ during SE. In support of this assumption, we have identified the RY- motif recognized by LEC2 (Braybrook et al., 2006) in the $P H B$ promoter (AGRIS Atcis DB) but the experimental verification for the direct binding of LEC2 to the $P H B$ promoter during SE would be necessary to test this further. Interestingly, the LEC2-binding RY-motif is also present in the promoters of the MIR165/166 genes (Wang and Perry, 2013) and thus it is conceivable that LEC2 might also control expression of MIR165/166 genes during SE. So far, few TFs directly regulating MIRNA genes have been implicated in plants including activation of MIR165a and MIR166b by SHR (SHORT ROOT) and SCR (SCARECROW) during postembryonic development (Carlsbecker et al., 2010; Miyashima et al., 2013). LEC2 regulation of MIRNA genes has not been reported yet but it cannot be excluded as FUS3, a TF structurally and functionally related with LEC2 (Harada, 2001), was suggested to control MIR156, MIR160, MIR166, MIR396 genes in the embryogenic culture of Arabidopsis (Wang and Perry, 2013).

Altogether, several lines of evidence infer that the miR165/166- $P H B / P H V$ regulatory node controls induction of the embryogenic program in somatic cells of Arabidopsis through targeting LEC2. The possible role of miR165/166 in the regulation of HD-ZIP III TFs during SE was also postulated in sweet orange and Larix leptolepis but the targeted effectors and molecular pathways controlled were not identified (Wu et al., 2011; Li et al., 2013).

Our results suggest that miR165/166- $P H B / P H V$ and miR160ARF10/ARF16 regulatory modules might regulate SE induction through LEC2. Accordingly, the significant changes in LEC2 expression levels in SE cultures with a disturbed expression and function of the ARF10 and ARF16 genes (MARF16 and arf10arf16) suggest that these ARFs positively regulate $L E C 2$. In addition, ARF10 and ARF16 seem to contribute to LEC2 regulation in SE due to the auxin-stimulated expression of $L E C 2$ and the similarity of spatio-temporal expression pattern of LEC2 and ARF10/ARF16 in embryogenic culture (Kurczynska et al., 2007; Ledwon and Gaj, 2009; Wójcikowska and Gaj, 2017). Considering that ARF10 and ARF16 repress auxin-regulated genes (Guilfoyle and Hagen, 2007) they are unlikely to control LEC2 directly and the intermediary genetic elements remain to be identified.

In conclusion, both the miR165/166- $P H B / P H V$ and miR160ARF10/ARF16 nodes might control the embryogenic transition in Arabidopsis somatic cells via regulating $L E C 2$, which is a key regulator of SE induction. The convergent functions of miR160 and miR165/166 in regulation of a common TF, the WOX5 (WUSCHEL-RELATED HOMEOBOX 5) gene, was reported in control of distal stem cell differentiation and embryonic root development (Grigg et al., 2009; Ding and Friml, 2010). Given that a role of WOX5 in formation of RAM in somatic embryos of Arabidopsis was reported (Su et al., 2015; Wang and Chong, 2016) the contribution of miR160 and miR165/166 to SE through regulation of the embryonic root development might be considered.

In addition to controlling the auxin biosynthesis-related $L E C 2$ gene, ARF10/ARF16 might also impact SE induction via the regulation of the genes that are involved in the signaling of other hormones including ABA. Accordingly, miR160-ARF10 was found to play an important role in $\mathrm{ABA}$-auxin crosstalk in seed germination and post-embryonic developmental programs (Liu et al., 2007). ARF16 was indicated to be required for the ABI3 expression (Liu et al., 2013) that encodes a transcription factor that is involved in ABA signaling during seed development (Finkelstein et al., 2002). It is worth noting that an abi3-1 mutant that was insensitive to ABA was found to be significantly impaired in its SE response (Gaj et al., 2006). These findings together with the extensive interactions between auxin and ABA signaling during plant development (Rock and Sun, 2005; Teale et al., 2008; Thole et al., 2013) including the induction of SE in Arabidopsis (Braybrook and Harada, 2008) infer that the disturbed ABA sensitivity that is expected in mARF16 might enhance auxin perception/signaling and as a result, an embryogenic response is triggered. Recently, the role of the ARF10-miR160 module in the regulation of cytokinin-auxin crosstalk was indicated (Liu et al., 2016). Important for the SEinduction mechanism, overexpression of miR160 was shown to enhance tissue sensitivity to cytokines (Turner et al., 2013), which were reported to play a key role in auxin-induced SE in carrot (Tokuji and Kuriyama, 2003) and Arabidopsis (Su et al., 2015; Wang and Chong, 2016).

The regulatory interaction between miR165/166 and miR160 in SE seems to include the negative feedback loop between ARF10/ARF16 and PHB/PHV, the targets of miR160 and miR165/166 pathways, respectively. Experimental supports for this notion include: (i) a positive impact of $P H B$ on the ARF10/ARF16 expression level (increased ARF10/ARF16 transcription in $p h b, p h v, p h b 1-d, S T T M 165 / 166$ (ii) a negative relation of ARF10/ARF16 on PHB transcription in the mARF16, $m i R 160 b$ and $m i R 160 c$ cultures. The higher expression of ARF10 and ARF16 in the STTM165/166 than in the phb1$d$ culture may be caused by an increased level of miR160, which is able to cleave ARF10/ARF16 transcripts (Wang et al., 2005) in phb1-d (Figure S6A). In support of a possible role of PHB in the direct activation of ARF10, ARF16 in SE, a binding of PHB to ARF5 promoter during vascular patterning in Arabidopsis was documented (Müller et al., 2015). However, it is also possible that ARF16 might repress $P H B$ via up-regulation of miR166 as we found a decreased level of miR166 in arf10arf16 culture (Figure S6B).

\section{CONCLUSIONS}

Our results indicate that miR160 and miR165/166-regulated pathways distinctly contribute to the regulation of developmental plasticity of Arabidopsis cells under in vitro conditions. Accordingly, miR160 and miR165/166 through targeting ARF10/ARF16 and PHB/PHV, respectively, were found to 
impact the SE induction through the LEC2-mediated auxinbiosynthesis pathway. In this scenario, the repression of both miR160 and miR165/166 leads to a higher expression of LEC2, which results in the YUC-mediated biosynthesis of auxin. As a consequence, IAA accumulates in explant tissues that trigger auxin responsive genes involved in the embryogenic transition.

Beside impacting the auxin biosynthesis miR165/166 might also contribute to the embryogenic transition via regulation of stress-related genes due to involvement of miR165/166 in modulation of abiotic stress responses (Jia et al., 2015) and a pivotal function of stress responses in SE induction mechanism (Zavattieri et al., 2010; Jin et al., 2014; Fehér, 2015). Thus, the SE-regulators might be also searched among the stress-related genes targeted by miR165/166.

This study provides a significant step forward in understanding the miRNA-mediated mechanism regulating developmental plasticity of plant somatic cells (Garcia, 2008; Rubio-Somoza and Weigel, 2011). The validation of the postulated regulatory interactions that act within and between the miR160- and miR165/166-regulated pathways and identification of other directly and indirectly controlled targets is essential to fully define the miRNA-mediated genetic network controlling SE induction.

\section{AUTHOR CONTRIBUTIONS}

MG and AW conceived and designed the research. AW conducted the experiments; MN provided the WISH of miRNA. MG and AW analyzed the data and wrote the manuscript. All the authors read and approved the manuscript.

\section{FUNDING}

This work was supported by a research grant from the National Science Centre in Poland (PRELUDIUM 2016/21/N/NZ2/01718).

\section{ACKNOWLEDGMENTS}

The authors wish to thank Prof. Xiaoya Chen (arf10-2arf16-2; pARF16::mARF16), Prof. Guiliang Tang (2x35S::STTM165/166), Prof. Keiji Nakajima ( $p P H B:: P H B m u-G F P$; $p P H B:: P H B m u-G F P$ ) for kindly providing seeds, and Magdalena Mosiolek for her technical advice and support in the implementation of the WISH procedure.

\section{REFERENCES}

Altamura, M. M., Rovere, F. D., Fattorini, L., D’Angeli, S., and Falasca, G. (2016). "Recent advances on genetic and physiological bases of in vitro somatic embryo formation," in In Vitro Embryogenesis in Higher Plants, Vol. 1359, ed M. A. Germana (New York, NY: Springer Science+Business Media), 47-86.

\section{SUPPLEMENTARY MATERIAL}

The Supplementary Material for this article can be found online at: https://www.frontiersin.org/articles/10.3389/fpls.2017. 02024/full\#supplementary-material

Figure S1 | The opposite expression patterns of miR165/166 vs. PHB, PHV (A) and miR160 vs. ARF10, ARF16, ARF17 (B) in the SE culture of WT (Col-0) according to the results for the expression level of miR160, miR165/166 (Szyrajew et al., 2017) and ARF10, ARF16, ARF17 (Wójcikowska and Gaj, 2017). Relative transcript level was normalized to the internal control (At4g27090) and calibrated to the 0 day of culture $(n=3)$; SE, somatic embryogenesis; d, day of SE culture.

Figure S2 | A negative control of WISH analysis of miRNA. A probe against mouse miR124 was used in analyses of WT (Col-0) explants cultured on SE-induction medium for 0 (A) 5 (B) and 10 (C) days; SE, somatic embryogenesis; $d$, day of SE culture.

Figure S3 | Expression profile of PHB and PHV in the SE culture of the phb and phv mutants that were induced on the medium with $5 \mu \mathrm{M}$ 2,4-D. The relative transcript level was normalized to the internal control (At4g27090) and calibrated to the WT culture. Statistical analyses were performed using two-way ANOVA $(P<0.05)$ followed by Tukey's honest significant difference test (Tukey HSD-test) $(P<0.05)$ in order to assess the differences between gene expression at 0,5 , and 10 days of the SE culture within a genotype and between genotypes. Statistically significant differences $(P<0.05)$ are indicated by different letters $(P<0.05 ; n=3$ \pm standard error). SE, somatic embryogenesis; d, day of SE culture.

Figure S4 | Expression level of the auxin-inducible IAA17 and IAA29 genes in the SE culture of the WT, miR160b, miR160c, mARF16, and STTM165/166 lines that were induced on the medium with $5 \mu \mathrm{M} 2,4-\mathrm{D}$. The relative transcript level was normalized to the internal control (At4g27090) and calibrated to 0 days of the WT culture. Statistical analyses were performed using two-way ANOVA $(P<0.05)$ followed by Tukey's honest significant difference test (Tukey HSD-test) $(P<0.05)$ in order to assess the differences between gene expression at 0,5 , and $10 \mathrm{~d}$ of the SE culture within a genotype and between genotypes. Statistically significant differences $(P<0.05)$ are indicated by different letters $(P<0.05 ; n=3 \pm$ standard error). SE, somatic embryogenesis; d, day of SE culture.

Figure S5 | Functional test of the arf10arf16, pPHB::muPHB-GFP, and phb1-d lines. The embryogenic potential of the arf10arf16, pPHB::muPHB-GFP (A) and phb1-d (B) lines and parental (WT) genotypes on the medium with $5 \mu$ M 2,4-D was measured by SE efficiency and SE productivity. Statistical analyses were performed using the $T$-test $(P<0.05)$ to assess the differences between the genotypes. Values that were significantly different from the WT culture are indicated with asterisks ( $n=3 \pm$ standard error).

Figure S6 | Expression level of miR160 (A) and miR166 (B) in the SE culture of the phb1-d, STTM165/166, and arf10arf16 transgenic lines that were induced on the medium with $5 \mu \mathrm{M} 2,4-\mathrm{D}$. The relative transcript level was normalized to the internal control (At4g27090) and calibrated to the WT culture. (A) Statistical analyses were performed using two-way ANOVA $(P<0.05)$ followed by Tukey's honest significant difference test (Tukey HSD-test) $(P<0.05)$ in order to assess the differences between the level of miR160 at 0,5 , and 10 days of the SE culture within a genotype and between genotypes. Statistically significant differences $(P<$ $0.05)$ are indicated by different letters $(P<0.05 ; n=3 \pm$ standard error). (B) Statistical analyses were performed using the $T$-test $(P<0.05)$ to assess the differences between the genotypes. Values that were significantly different from the WT-derived culture are indicated with an asterisk ( $n=3 \pm$ standard error).

Bedzhov, I., Graham, S. J. L., Leung, C. Y., and Zernicka-Goetz, M. (2014). Developmental plasticity, cell fate specification and morphogenesis in the early mouse embryo. Philos. Trans. R. Soc. B Biol. Sci. 369:20130538. doi: 10.1098/rstb.2013.0538

Boutilier, K., Offringa, R., Sharma, V. K., Kieft, H., Ouellet, T., and Zhang, L. (2002). Ectopic expression of BABY BOOM triggers a conversion 
from vegetative to embryonic growth. Plant Cell 14, 1737-1749. doi: $10.1105 /$ tpc. 001941

Braybrook, S. A., and Harada, J. J. (2008). LECs go crazy in embryo development. Trends Plant Sci. 13, 624-630. doi: 10.1016/j.tplants.2008.09.008

Braybrook, S. A., Stone, S. L., Park, S., Bui, A. Q., Le, B. H., Fischer, R. L., et al. (2006). Genes directly regulated by LEAFY COTYLEDON2 provide insight into the control of embryo maturation and somatic embryogenesis. Proc. Natl. Acad. Sci. U.S.A. 103, 3468-3473. doi: 10.1073/pnas.0511331103

Bric, J. M., Bostock, R. M., Silverstone, S. E.,(1991). Rapid in situ assay for indoleacetic acid production by bacteria immobilized on a nitrocellulose membrane rapid in situ assay for indoleacetic acid production by bacteria immobilized on a nitrocellulose membrane. Appl. Environ. Microbiol. 57, 535-538.

Bustos-Sanmamed, P., Mao, G., Deng, Y., Elouet, M., Khan, G. A., Bazin, J., et al. (2013). Overexpression of miR160 affects root growth and nitrogenfixing nodule number in Medicago truncatula. Funct. Plant Biol. 40, 1208-1220. doi: 10.1071/FP13123

Carlsbecker, A., Lee, J.-Y., Roberts, C. J., Dettmer, J., Lehesranta, S., Zhou, J., et al. (2010). Cell signalling by microRNA165/6 directs gene dose-dependent root cell fate. Nature 465, 316-321. doi: 10.1038/nature08977

Chávez-Hernández, E. C., Alejandri-Ramírez, N. D., Juárez-González, V. T., and Dinkova, T. D. (2015). Maize miRNA and target regulation in response to hormone depletion and light exposure during somatic embryogenesis. Front. Plant Sci. 6:555. doi: 10.3389/fpls.2015.00555

Chen, C.-J., Liu, Q., Zhang, Y.-C., Qu, L.-H., Chen, Y.-Q., and Gautheret, D. (2011). Genome-wide discovery and analysis of microRNAs and other small RNAs from rice embryogenic callus. RNA Biol. 8, 538-547. doi: 10.4161/rna.8.3.15199

Clough, S. J., and Bent, A. F. (1998). Floral dip: a simplified method for Agrobacterium-mediated transformation of Arabidopsis thaliana. Plant J. 16, 735-743. doi: 10.1046/j.1365-313x.1998.00343.x

Damodharan, S., Zhao, D., and Arazi, T. (2016). A common miRNA160-based mechanism regulates ovary patterning, floral organ abscission and lamina out growth in tomato. Plant J. 86, 458-471. doi: 10.1111/tpj.13127

Dastidar, M. G., Mosiolek, M., Bleckmann, A., Dresselhaus, T., Nodine, M. D., and Maizel, A. (2016). Sensitive whole mount in situ localization of small RNAs in plants. Plant J. 88, 694-702. doi: 10.1101/037978

Ding, Z., and Friml, J. (2010). Auxin regulates distal stem cell differentiation in Arabidopsis roots. Proc. Natl. Acad. Sci. U.S.A. 107, 12046-12051. doi: $10.1073 /$ pnas. 1000672107

Du, Q., and Wang, H. (2015). The role of HD-ZIP III transcription factors and miR165/166 in vascular development and secondary cell wall formation. Plant Signal. Behav. 10:e1078955. doi: 10.1080/15592324.2015.1078955

Elithi, M., and Stasolla, C. (2011). "The use of zygotic embryos as explants for in vitro propagation: an overview," in Plant Embryo Culture: Methods and Protocols, Vol. 710, eds T. A. Thorpe and E. C. Yeung (New Delhi: Springer Science+Business Media), 229-255.

Fehér, A. (2015). Somatic embryogenesis - stress-induced remodeling of plant cell fate. Biochim. Biophys. Acta 1849, 385-402. doi: 10.1016/j.bbagrm.2014.07.005

Finkelstein, R. R., Gampala, S. S., and Rock, C. D. (2002). Abscisic acid signaling in seeds and seedlings. Plant Cell. 14, S15-S45. doi: 10.1105/tpc.010441

Gaj, M. D. (2001). Direct somatic embryogenesis as a rapid and efficient system for in vitro regeneration of Arabidopsis thaliana. Plant Cell Tissue Organ Cult. 64, 39-46. doi: 10.1023/A:1010679614721

Gaj, M. D. (2004). Factors influencing somatic embryogenesis induction and plant regeneration with particular reference to Arabidopsis. thaliana (L.) Heynh. Plant Growth Regul. 43, 27-47. doi: 10.1023/B:GROW.0000038275.29262.fb

Gaj, M. D., Trojanowska, A., Ujczak, A., Medrek, M., Kozioł, A., and Garbaciak, B. (2006). Hormone-response mutants of Arabidopsis thaliana (L.) Heynh. impaired in somatic embryogenesis. Plant Growth Regul. 49, 183-197. doi: 10.1007/s10725-006-9104-8

Gaj, M. D., Zhang, S., Harada, J. J., and Lemaux, P. G. (2005). LEAFY COTYLEDON genes are essential for induction of somatic embryogenesis of Arabidopsis. Planta 222, 977-988. doi: 10.1007/s00425-005-0041-y

Garcia, D. (2008). A miRacle in plant development: role of microRNAs in cell differentiation and patterning. Semin. Cell Dev. Biol. 19, 586-595. doi: $10.1016 / j . s e m c d b .2008 .07 .013$
Gliwicka, M., Nowak, K., Balazadeh, S., Mueller-Roeber, B., and Gaj, M. D. (2013). Extensive modulation of the transcription factor transcriptome during somatic embryogenesis in Arabidopsis thaliana. PLoS ONE 8:e69261. doi: 10.1371/journal.pone.0069261

Grigg, S. P., Galinha, C., Kornet, N., Canales, C., Scheres, B., and Tsiantis, M. (2009). Report repression of apical homeobox genes is required for embryonic root development in Arabidopsis. Curr. Biol. 19, 1485-1490. doi: 10.1016/j.cub.2009.06.070

Guilfoyle, T. J., and Hagen, G. (2007). Auxin response factors. Curr. Opin. Plant Biol. 10, 453-460. doi: 10.1016/j.pbi.2007.08.014

Gutierrez, L., Bussell, J. D., Pacurar, D. I., Schwambach, J., Pacurar, M., and Bellini, C. (2009). Phenotypic plasticity of adventitious rooting in Arabidopsis is controlled by complex regulation of AUXIN RESPONSE FACTOR transcripts and microRNA abundance. Plant Cell 21, 3119-3132. doi: $10.1105 /$ tpc. 108.064758

Harada, J. J. (2001). Role of Arabidopsis LEAFY COTYLEDON genes in seed development. J. Plant Physiol. 158, 405-409. doi: 10.1078/0176-1617-00351

Harding, E. W., Tang, W., Nichols, K. W., Fernandez, D. E., and Perry, S. E. (2003). Expression and maintenance of embryogenic potential is enhanced through constitutive expression of AGAMOUS-Like 15. Plant Physiol. 133, 653-663. doi: 10.1104/pp.103.023499

Jefferson, R. A., Kavanagh, T. A., and Bevan, M. W. (1987). GUS fusions: beta glucuronidase as a sensitive and versatile gene fusion marker in higher plants. EMBO J. 6, 3901-3907.

Jeong, S., Volny, M., and Lukowitz, W. (2012). Axis formation in Arabidopsis transcription factors tell their side of the story. Curr. Opin. Plant Biol. 15, 4-9. doi: 10.1016/j.pbi.2011.10.007

Jia, H., Suzuki, M., and McCarty, D. R. (2014). Regulation of the seed to seedling developmental phase transition by the LAFL and VAL transcription factor networks. Wiley Interdiscip. Rev. Dev. Biol. 3, 135-145. doi: 10.1002/wdev.126

Jia, X., Ding, N., Fan, W., Yan, J., Gu, Y., Tang, X., et al. (2015). Functional plasticity of miR165/166 in plant development revealed by small tandem target mimic. Plant Sci. 233, 11-21. doi: 10.1016/j.plantsci.2014. 12.020

Jin, F., Hu, L., Yuan, D., Xu, J., Gao, W., He, L., et al. (2014). Comparative transcriptome analysis between somatic embryos (SEs) and zygotic embryos in cotton : evidence for stress response functions in SE development. Plant Biotechnol. J. 12, 161-173. doi: 10.1111/pbi.12123

Jones-Rhoades, M. W., Bartel, D. P., and Bartel, B. (2006). MicroRNAs and their regulatory roles in plants. Annu. Plant Biol. 57, 19-53. doi: 10.1146/annurev.arplant.57.032905.105218

Jung, J. H., and Park, C. M. (2007). MIR166/165 genes exhibit dynamic expression patterns in regulating shoot apical meristem and floral development in Arabidopsis. Planta 225, 1327-1338. doi: 10.1007/s00425-006-0439-1

Kurczynska, E. U., Gaj, M. D., Ujczak, A., and Mazur, E. (2007). Histological analysis of direct somatic embryogenesis in Arabidopsis thaliana (L.) Heynh. Planta 226, 619-628. doi: 10.1007/s00425-007-0510-6

Lai, X., Wolkenhauer, O., and Vera, J. (2016). Understanding microRNA-mediated gene regulatory networks through mathematical modelling. Nucleic Acids Res. 44, 6019-6035. doi: 10.1093/nar/gkw550

Lara-Chavez, A., Egertsdotter, U., and Flinn, B. S. (2012). Comparison of gene expression markers during zygotic and somatic embryogenesis in pine. In Vitro Cell. Dev. Biol. 48, 341-354. doi: 10.1007/s11627-012-9440-5

Le, B. H., Cheng, C., Bui, A. Q., Wagmaister, J., a, Henry, K. F., Pelletier, J., et al. (2010). Global analysis of gene activity during Arabidopsis seed development and identification of seed-specific transcription factors. Proc. Natl. Acad. Sci. U.S.A. 107, 8063-8070. doi: 10.1073/pnas. 1003530107

Ledwon, A., and Gaj, M. D. (2009). LEAFY COTYLEDON2 gene expression and auxin treatment in relation to embryogenic capacity of Arabidopsis somatic cells. Plant Cell Rep. 28:1677. doi: 10.1007/s00299-009-0767-2

Ledwon, A., and Gaj, M. D. (2011). LEAFY COTYLEDON1, FUSCA3 expression and auxin treatment in relation to somatic embryogenesis induction in Arabidopsis. Plant Growth Regul. 65, 157-167. doi: 10.1007/s10725-011-9585-y

Li, S. G., Li, W. F., Han, S. Y., Yang, W. H., and Qi, L. W. (2013). Stagespecific regulation of four HD-ZIP III transcription factors during polar pattern formation in Larix leptolepis somatic embryos. Gene 522, 177-183. doi: 10.1016/j.gene.2013.03.117 
Li, T., Chen, J., Qiu, S., Zhang, Y., Wang, P., Yang, L., et al. (2012). Deep sequencing and microarray hybridization identify conserved and speciesspecific microRNAs during somatic embryogenesis in hybrid yellow poplar. PLoS ONE 7:e43451. doi: 10.1371/journal.pone.0043451

Lin, Y., and Lai, Z. (2013). Comparative analysis reveals dynamic changes in miRNAs and their targets and expression during somatic embryogenesis in longan (Dimocarpus longan Lour.). PLoS ONE 8:e60337. doi: 10.1371/journal.pone.0060337

Lin, Y., Lai, Z., Tian, Q., Lin, L., Lai, R., Yang, M., et al. (2015). Endogenous target mimics down-regulate miR160 mediation of ARF10,-16, and-17 cleavage during somatic embryogenesis in Dimocarpus longan Lour. Mol. Breed. 35, 1-16. doi: 10.1007/s11032-015-0420-4

Liu, P. P., Montgomery, T. A., Fahlgren, N., Kasschau, K. D., Nonogaki, H., and Carrington, J. C. (2007). Repression of AUXIN RESPONSE FACTOR10 by microRNA160 is critical for seed germination and post-germination stages. Plant J. 52, 133-146. doi: 10.1111/j.1365-313X.2007.03218.x

Liu, X., Huang, J., Wang, Y., Khanna, K., Xie, Z., Owen, H. A., et al. (2010). The role of floral organs in carpels, an Arabidopsis loss-of-function mutation in MicroRNA160a, in organogenesis and the mechanism regulating its expression. Plant J. 62, 416-428. doi: 10.1111/j.1365-313X.2010.04164.X

Liu, X., Zhang, H., Zhao, Y., Feng, Z., Li, Q., Yang, H. Q., et al. (2013). Auxin controls seed dormancy through stimulation of abscisic acid signaling by inducing ARF-mediated ABI3 activation in Arabidopsis. Proc. Natl. Acad. Sci. U.S.A. 17, 15485-15490. doi: 10.1073/pnas.1304651110

Liu, Z., Li, J., Wang, L., Li, Q., Lu, Q., Yu, Y., et al. (2016). Repression of callus initiation by the miRNA-directed interaction of auxin-cytokinin in Arabidopsis thaliana. Plant J. 4, 391-402. doi: 10.1111/tpj.13211

Mallory, A. C., Bartel, D. P., and Bartel, B. (2005). MicroRNA-directed regulation of Arabidopsis AUXIN RESPONSE FACTOR17 is essential for proper development and modulates expression of early auxin response genes. Development 17, 1-16. doi: 10.1105/tpc.105.031716

Mallory, A. C., Reinhart, B. J., Jones-Rhoades, M. W., Tang, G., Zamore, P. D., Barton, M. K., et al. (2004). MicroRNA control of PHABULOSA in leaf development: importance of pairing to the microRNA 5' region. EMBO J. 23, 3356-3364. doi: 10.1038/sj.emboj.7600340

McConnell, J. R., and Barton, M. K. (1998). Leaf polarity and meristem formation in Arabidopsis. Development 125, 2935-2942.

McConnell, J. R., Emery, J., Eshed, Y., Bao, N., Bowman, J., and Barton, M. K. (2001). Role of PHABULOSA and PHAVOLUTA in determining radial patterning in shoots. Nature 411, 709-713. doi: 10.1038/35079635

Misra, P., and Saema, S. (2016). "Plant tissue culture for in vitro mutagenesis, large-scale propagation, and genetic transformation," in Plant Tissue Culture: Propagation, Conservation and Crop Improvement, eds M. Anis and N. Ahmad (Singapore: Springer Singapore), 309-342.

Miyashima, S., Honda, M., Hashimoto, K., Tatematsu, K., Hashimoto, T., SatoNara, K., et al. (2013). A comprehensive expression analysis of the Arabidopsis MICRORNA165/6 gene family during embryogenesis reveals a conserved role in meristem specification and a non-cell-autonomous function. Plant Cell Physiol. 54, 375-384. doi: 10.1093/pcp/pcs188

Miyashima, S., Koi, S., Hashimoto, T., and Nakajima, K. (2011). Non-cellautonomous microRNA165 acts in a dose-dependent manner to regulate multiple differentiation status in the Arabidopsis root. Development 138, 2303-2313. doi: 10.1242/dev.060491

Müller, C. J., Valdés, A. E., Wang, G., Ramachandran, P., Beste, L., Uddenberg, D., et al. (2015). PHABULOSA mediates an auxin signaling loop to regulate vascular patterning in Arabidopsis. Plant Physiol. 170, 956-970. doi: 10.1104/pp.15.01204

Nodine, M. D., and Bartel, D. P. (2010). MicroRNAs prevent precocious gene expression and enable pattern formation during plant embryogenesis. Genes Dev. 24, 2678-2692. doi: 10.1101/gad.1986710

Nowak, K., and Gaj, M. (2016). Transcription factors in the regulation of somatic embryogenesis," in Somatic Embryogenesis: Fundamental Aspects and Applications, eds V. M. Loyola-Vargas and N. Ochoa-Alejo (Springer International Publishing), 53-79.

Overvoorde, P., Okushima, Y., Alonso, J., Chan, A., Chang, C., Ecker, J. R., et al. (2005). Functional genomic analysis of the AUXIN/INDOLE-3-ACETIC ACID gene family members in Arabidopsis thaliana. Plant Cell 17, 3282-3300. doi: $10.1105 /$ tpc. 105.036723
Prigge, M. J., Otsuga, D., Alonso, J. M., Ecker, J. R., Drews, G. N., and Clark, S. E. (2005). Class III homeodomain-leucine zipper gene family members have overlapping, antagonistic, and distinct roles in Arabidopsis development. Plant Cell 17, 61-76. doi: 10.1105/tpc.104.026161

Reinhart, B. J., Weinstein, E. G., Rhoades, M. W., Bartel, B., and Bartel, D. P. (2002). MicroRNAs in plants. Genes Dev. 13, 1616-1626. doi: $10.1101 / \mathrm{gad} .1004402$

Rock, C. D., and Sun, X. (2005). Crosstalk between ABA and auxin signaling pathways in roots of Arabidopsis thaliana (L.) Heynh. Planta 222, 98-106. doi: $10.1007 / \mathrm{s} 00425-005-1521-9$

Rubio-Somoza, I., and Weigel, D. (2011). MicroRNA networks and developmental plasticity in plants. Trends Plant Sci. 16, 258-264. doi: 10.1016/j.tplants.2011.03.001

Seefried, W. F., Willmann, M. R. R., Clausen, R. L., and Jenik, P. D. (2014). Global regulation of embryonic patterning in Arabidopsis by microRNAs. Plant Physiol. 165, 670-687. doi: 10.1104/pp.114.240846

Shi, Z., Zhang, C., Xu, X., Zhu, J., Zhou, Q., Ma, L., et al. (2015). Overexpression of AtTTP affects ARF17 expression and leads to male sterility in Arabidopsis. PLOS ONE 10:e0117317. doi: 10.1371/journal.pone.0117317

Sieber, P., Gheyselinck, J., Gross-Hardt, R., Laux, T., Grossniklaus, U., and Schneitz, K. (2004). Pattern formation during early ovule development in Arabidopsis thaliana. Dev. Biol. 273, 321-334. doi: 10.1016/j.ydbio.2004.05.037

Smith, Z. R., and Long, J. (2010). Control of Arabidopsis apical-basal embryo polarity by antagonistic transcription factors. Nature 464, 423-426. doi: $10.1038 /$ nature 08843

Speth, C., and Laubinger, S. (2014). "Rapid and parallel quantification of small and large RNA species," in Plant Circadian Networks: Methods and Protocols, ed D. Staiger (New York, NY: Springer New York), 93-106.

Stone, S. L., Kwong, L. W., Yee, K. M., Pelletier, J., Lepiniec, L., Fischer, R. L., et al. (2001). LEAFY COTYLEDON2 encodes a B3 domain transcription factor that induces embryo development. Proc. Natl. Acad. Sci. U.S.A. 98, 11806-11811. doi: 10.1073/pnas.201413498

Su, Y. H., Liu, Y. B., Bai, B., and Zhang, X. S. (2015). Establishment of embryonic shoot-root axis is involved in auxin and cytokinin response during Arabidopsis somatic embryogenesis. Front. Plant Sci. 5:792. doi: 10.3389/fpls.2014. 00792

Szyrajew, K., Bielewicz, D., Dolata, J., Wójcik, A. M., Nowak, K., SzczygiełSommer, A., Szweykowska-Kulinska, Z., et al. (2017). MicroRNAs are intensively regulated during induction of somatic embryogenesis in Arabidopsis. Front Plant Sci. 8:18. doi: 10.3389/fpls.2017.00018

Tang, G., Reinhart, B. J., Bartel, D. P., and Zamore, P. D. (2003). A biochemical framework for RNA silencing in plants A biochemical framework for RNA silencing in plants. Genes Dev. 2, 49-63. doi: 10.1101/gad.1048103

Tang, X., Bian, S., Tang, M., Lu, Q., Li, S., Liu, X., et al. (2012). MicroRNA-mediated repression of the seed maturation program during vegetative development in Arabidopsis. PLoS Genet. 8:e1003091. doi: 10.1371/journal.pgen.1003091

Teale, W. D., Ditengou, F. A., Dovzhenko, A. D., Li, X., Molendijk, A. M., Ruperti, B., et al. (2008). Auxin as a model for the integration of hormonal signal processing and transduction. Mol. Plant 1, 229-237. doi: 10.1093/mp/ ssn006

Thellin, O., and Zorzi, W. (1999). Housekeeping genes as internal standards: use and limits. J. Biotechnol. 75, 291-295. doi: 10.1016/S0168-1656(99)00163-7

Thole, J. M., Beisner, E. R., Liu, J., Venkova, S., and Strader, L. C. (2013). Abscisic Acid regulates root elongation through the activities of auxin and ethylene in Arabidopsis thaliana. G3 (Bethesda). 15, 1259-1274. doi: 10.1534/g3.114.011080

Tokuji, Y., and Kuriyama, K. (2003). Involvement of gibberellin and cytokinin in the formation of embryogenic cell clumps in carrot (Daucus carota). J. Plant Physiol. 160. 133-141 doi: 10.1078/0176-1617-00892

Turner, M., Nizampatnam, N. R., Baron, M., Coppin, S., Damodaran, S., Adhikari S., et al. (2013). Ectopic expression of miR160 results in auxin hypersensitivity, cytokinin hyposensitivity, and inhibition of symbiotic nodule development in soybean. Plant Physiol. 162, 2042-2055. doi: 10.1104/pp.113.220699

Wang, F., and Perry, S. E. (2013). Identification of Direct Targets of FUSCA3, a key regulator of Arabidopsis seed development. Plant Physiol. 161, 1251-1264. doi: 10.1104/pp.112.212282

Wang, J. W., Wang, L. J., Mao, Y. B., Cai, W. J., Xue, H. W., and Chen, X. Y. (2005). Control of root cap formation by microRNA-targeted auxin response factors in Arabidopsis. Plant Cell Online 17, 2204-2216. doi: 10.1105/tpc.105.033076 
Wang, L., and Chong, K. (2016). The essential role of cytokinin signaling in root apical meristem formation during somatic embryogenesis. Front. Plant Sci. 6:1196. doi: 10.3389/fpls.2015.01196

Wójcik, A. M., and Gaj, M. D. (2016). miR393 contributes to the embryogenic transition induced in vitro in Arabidopsis via the modification of the tissue sensitivity to auxin treatment. Planta 244, 231-243. doi: 10.1007/s00425-016-2505-7

Wójcikowska, B., and Gaj, M. D. (2016). "Somatic embryogenesis in Arabidopsis," in Somatic Embryogenesis: Fundamental Aspects and Applications, eds V. M. Loyola-Vargas and N. Ochoa-Alejo (Springer International Publishing), 185-199.

Wójcikowska, B., and Gaj, M. D. (2017). Expression profiling of AUXIN RESPONSE FACTOR genes during somatic embryogenesis induction in Arabidopsis. Plant Cell Rep. 36, 843-858. doi: 10.1007/s00299-017-2114-3

Wójcikowska, B., Jaskóła, K., Gasiorek, P., Meus, M., Nowak, K., and Gaj, M. D. (2013). LEAFY COTYLEDON2 (LEC2) promotes embryogenic induction in somatic tissues of Arabidopsis, via YUCCA-mediated auxin biosynthesis. Planta 238, 425-440. doi: 10.1007/s00425-013-1892-2

Wu, G., Park, M. Y., Conway, S. R., Wang, J., Weigel, D., and Scott, R. (2009). The sequential actions of miR156 and miR172 regulates developmental timing in Arabidopsis. Cell 138, 750-759. doi: 10.1016/j.cell.2009.06.031

Wu, X. M., Liu, M. Y., Ge, X. X., Xu, Q., and Guo, W. W. (2011). Stage and tissue-specific modulation of ten conserved miRNAs and their targets during somatic embryogenesis of Valencia sweet orange. Planta 233, 495-505. doi: 10.1007/s00425-010-1312-9

Xiang, D., Venglat, P., Tibiche, C., Yang, H., Risseeuw, E., Cao, Y., et al. (2011). Genome-wide analysis reveals gene expression and metabolic network dynamics during embryo development in Arabidopsis. Plant Physiol. 156, 346-356. doi: 10.1104/pp.110.171702

Yan, J., Gu, Y., Jia, X., Kang, W., Pan, S., Tang, X., et al. (2012). Effective small RNA destruction by the expression of a short tandem target mimic in Arabidopsis. Plant Cell 24, 415-427. doi: 10.1105/tpc.111.094144
Yang, J., Tian, L., Sun, M.-X., Huang, X.-Y., Zhu, J., Guan, Y.-F., et al. (2013) AUXIN RESPONSE FACTOR17 is essential for pollen wall pattern formation in Arabidopsis. Plant Physiol. 162, 720-731. doi: 10.1104/pp.113.214940

Ye, Y., Gong, Z., Lu, X., Miao, D., Shi, J., Lu, J., et al. (2016). Germostatin resistance locus 1 encodes a PHD finger protein involved in auxinmediated seed dormancy and germination. Plant J. 85, 3-15. doi: 10.1111/tpj. 13086

Zavattieri, M. A., Frederico, A. M., Lima, M., Sabino, R., and ArnholdtSchmitt, B. (2010). Induction of somatic embryogenesis as an example of stress-related plant reactions. Electron. J. Biotechnol. 13, 1-9. doi: 10.2225/vol13-issue1-fulltext-4

Zhang, J., Zhang, S., Han, S., Wu, T., Li, X., Li, W., et al. (2012). Genomewide identification of microRNAs in larch and stage-specific modulation of 11 conserved microRNAs and their targets during somatic embryogenesis. Planta 236, 647-657. doi: 10.1007/s00425-012-1643-9

Zhong, R., and Ye, Z.-H. (2007). Regulation of HD-ZIP III genes by microRNA 165. Plant Signal. Behav. 5, 351-353. doi: 10.4161/psb.2.5.4119

Zuo, J., Niu, Q., Frugis, G., and Chua, N. (2002). The WUSCHEL gene promotes vegetative-to-embryonic transition in Arabidopsis. Plant J. 30, 349-359. doi: 10.1046/j.1365-313X.2002.01289.x

Conflict of Interest Statement: The authors declare that the research was conducted in the absence of any commercial or financial relationships that could be construed as a potential conflict of interest.

Copyright (c) 2017 Wójcik, Nodine and Gaj. This is an open-access article distributed under the terms of the Creative Commons Attribution License (CC BY). The use, distribution or reproduction in other forums is permitted, provided the original author(s) or licensor are credited and that the original publication in this journal is cited, in accordance with accepted academic practice. No use, distribution or reproduction is permitted which does not comply with these terms. 